\title{
3 Systemgestaltung als professionelles Handeln
}

\subsection{Einführung}

In diesem Kapitel wird näher dargelegt, was unter Systemgestaltung verstanden wird. Es wird aufgezeigt, welche Kontextbedingungen dieses spezifische Handeln prägen (Abschnitt 3.2), welche Theorien und Konzepte bedeutsam sind (Abschnitt 3.3) und welches professionelle Handlungsverständnis die Autoren bei der Systemgestaltung respektive in der Sozialplanung als wichtig erachten (Abschnitt 3.5). Im Zentrum steht die Frage, welche Handlungskompetenzen für eine professionelle Systemgestaltung in der Sozialwirtschaft benötigt werden (Abschnitt 3.4).

Damit liegt der Blick auf dem Element (b) des Luzerner Gestaltungsmodells (LUS): der 'Systemgestaltung'. Der Begriff «Systemgestaltung» ist neu; häufiger sind die Bezeichnungen «Planung» oder «Steuerung» zu finden. Die Terminologie von Planen und Steuern wird sehr oft technizistisch verstanden und damit der Glaube an die Steuerbarkeit sozialer Systeme und die «Machbarkeit» von Planungsprozessen verstärkt. Die Autoren sind einerseits überzeugt, dass das Planen von Prozessen wichtig ist, andererseits ist es ihnen aber auch bewusst, dass die vielfältigen Rahmenbedingungen und Einflussfaktoren sowie die Mitbeteiligung verschiedener Akteure bei der sozialpolitischen Planung den Prozessverlauf ebenso mitbestimmen - und dies zu Recht -, sodass die «Kunst» des Planens und Steuerns eher als ein Gestalten verstanden werden kann. Damit distanzieren sich die Autoren von einem linearen Verständnis von Handeln und haben mit dem Begriff des Gestaltens ein Wort gewählt, das vor allem auch Handeln als etwas Interaktives, Prozessbezogenes und Partizipatives versteht. Sie sind sich nicht nur theoretisch, sondern auch praktisch der Grenzen der Steuerbarkeit von komplexen Versorgungssystemen bewusst. Auch wenn vom Gegenstand her gedacht das Gleiche gemeint ist wie bei der Steuerung und Planung, so soll das dem Begriff zugrundeliegende Handlungsverständnis mit dem Begriff des Gestaltens in besonderem Mass verdeutlichet werden. Doch was steckt hinter dem Begriff des Gestaltens? Welche methodischen Kompetenzen sind damit verbunden?

Methodisches Handeln ist für die Beratung von Klientinnen und Klienten, Familien und Gruppen - und damit in Kleinsystemen - eine Kernkompetenz der Sozialen Arbeit. Professionelle Interventionen in grösseren Systemen kommen in der Gemeinwesenarbeit, der soziokulturellen Animation, der Organisationsentwicklung und im Sozialmanagement vor. Wenn hier von Systemgestaltung ge- 
sprochen wird, wird sich einerseits an den klassischen Konzepten der Handlungslehre der Sozialen Arbeit orientiert (Galuske 2013, Heiner 2010, von Spiegel 2013), die durch einen systemischen Ansatz erweitert wurden (z.B. Hosemann/Geiling 2013). Andererseits versuchen die Autoren, diese Konzepte, die auf Kleinsysteme ausgerichtet sind, auf die Gestaltung und Steuerung von Versorgungssystemen zu übertragen und nehmen auch Bezug auf aktuelle Publikationen zur Sozialplanung (Böhmer 2014, Reichwein/Berg/Glasen/Junker/Rottler-Nourbakhsch/Vogel 2011).

Es würde allerdings den Rahmen dieses Buchs sprengen, wenn dessen Autoren in diesem Zusammenhang auf den umfangreichen Diskurs zum Thema des methodischen Handelns eingehen würden. Es geht in erster Linie darum, das Handeln in grossen und komplexen Versorgungssystemen in den Grundzügen zu umreissen. Es handelt sich dabei um eine Gestaltungsrolle, die verschiedene Kompetenzen voraussetzt.

Welches sind nun diese Kernkompetenzen? Auf dieser Suche sind die Autoren von Einflussfaktoren ausgegangen, die aus ihrer Sicht professionelles Handeln prägen können. In diesem Zusammenhang zu nennen wären diesbezüglich unter anderem

- gesellschaftliche, politische oder ökonomische Entwicklungen, welche die Systemgestaltung beeinflussen (Abschnitte 3.2.1 bis 3.2.3);

- $\quad$ strukturelle Rahmendigungen, welche die institutionelle Landschaft prägen, wie zum Beispiel die Aufgabenteilung zwischen Bund, Kantonen und Gemeinden, gesetzliche Bestimmungen oder die Aufgabenteilung zwischen staatlichen Organen und privaten Trägerschaften (Abschnitte 3.2.4 und 3.2.5);

- $\quad$ professionsspezifische Theorien und Konzepte, die einer Systemgestaltung dienen und die beigezogen werden können oder die einschlägige (Handlungs-) Anforderungen definieren (Abschnitte 3.3.1 bis 3.3.6).

Aus der Beschreibung und Analyse dieser Einflussgrössen wurden fünf zentrale Handlungskompetenzen für die Systemgestaltung abgeleitet (Abschnitt 3.4):

(1) Sozialwirtschaftskompetenz: In dieser Handlungskompetenz verbindet sich eine fundierte Branchenkenntnis der Sozialwirtschaft mit ökonomischen Kernkompetenzen.

(2) Systemverständnis und Systemübersetzung: Angesichts der Komplexität und potenzieller Widersprüchlichkeiten bei gleichzeitigen Abhängigkeiten erscheinen die Sensitivität und Empathie für unterschiedliche Systemrealitäten und -logiken sowie eine hohe Übersetzungs- und Vermittlungskompetenz in 
der Kommunikation zwischen den unterschiedlich ausgerichteten Systemen ausserordentlich bedeutsam.

(3) Konzeptualisierungs- und Entwicklungskompetenz: Diese Handlungskompetenz umfasst vorausschauendes, strategisches sowie innovatives, zukunftsorientiertes Denken und analytische Fähigkeiten, um entsprechende Fachkonzepte zur Gestaltung in Versorgungssystemen zu entwickeln und zu verfassen.

(4) Vernetzungs- und Kooperationskompetenz: Diese Kompetenz umfasst die Fach- und Methodenkompetenz, verschiedenste Akteure mit ihren unterschiedlichsten Anliegen, Interessen und Leistungskapazitäten in produktive und verbindliche Formen der Zusammenarbeit zu bringen.

(5) Prozesskompetenz und Fähigkeit zum Projektmanagement: Diese Kompetenz beinhaltet die Fähigkeit, ein zielgerichtetes Projektmanagement durchzuführen, das unterschiedlichsten Erwartungen von Akteuren sowie Systemlogiken gerecht wird und die Prozessgestaltung laufend darauf ausrichtet.

Handlungskompetenzen sind unbestrittene Kernelemente professionellen Handelns. Im Verständnis der Buchautoren ist Professionalität ferner durch bestimmte Werte und Haltungen geprägt, die das Handeln auszeichnen. Daher schliesst dieses Kapitel (Abschnitt 3.5) mit einer Beschreibung von ausgewählten Werten und Haltungen, die die Autoren für die Systemgestaltung für besonders nützlich und relevant halten. Aus dieser Sicht gehören der Umgang mit Dilemmata, eine permanente Reflexion des eigenen Handelns und eine hohe Prozessorientierung dazu. Abbildung 7 stellt den Inhalt und Aufbau dieses Kapitels in einer schematischen Übersicht dar. 
Kontextbedingungen der professionellen Systemgestaltung (3.2)

3.2.1 Sozialpolitische Rahmenbedingungen in der Schweiz

3.2.2 Finanzielle Rahmenbedingungen

3.2.3 Ökonomisierung der Sozialen Arbeit

3.2.4 Welfare-Mix

3.2.5 DifferenzvonSozial-und Verwaltungsraum
Theoriebezüge ausgewählter

Handlungskonzepte (3.3)

3.3.1 Theorieentwicklung zu Sozialmanagement und Sozialwirtschaft

3.3.2 Management und Governance

3.3.3 Organisationales Handeln

3.3.4 Kooperationsformen

3.3.5 Innovation

3.3.6 Qualitätsentwicklung

\section{$\frac{7}{7}$}

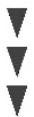

Zentrale Handlungskompetenzen in der Systemgestaltung (3.4)

3.4.1 Sozialwirtschaftskompetenz

3.4.2 Systemverständnis und Systemübersetzung

3.4.3 Konzeptualisierungs- und Entwicklungskompetenz

3.4.4 Vernetzungs- und Kooperationskompetenz

3.4.5 Prozesskompetenz und Projektmanagement

Professionelles Handlungsverständnis (3.5)

3.5.1 Der Gestaltungsbegriff

3.5.2 Reflexive Professionalität

3.5.3 Partizipation

3.5.4 Prozessorientierung

3.5.5 Umgang mit Spannungsfeldern und Dilemmata

Abbildung 7: Aufbau des Kapitels 3

Quelle: eigene Darstellung 


\subsection{Kontextbedingungen der professionellen Systemgestaltung}

Die Systemgestaltung in der Sozialwirtschaft ist wesentlich von ihrem Umfeld geprägt. Diesbezüglich zu nennen sind politische und finanzielle Rahmenbedingungen, die Ökonomisierung der Sozialen Arbeit oder der Mix sozialstaatlicher Leistungen und unterschiedlicher Leistungserbringender. In den folgenden Abschnitten werden relevante Kontextbedingungen vorgestellt und daraus zentrale Kompetenzen für die Systemgestaltung abgeleitet.

\subsubsection{Sozialpolitische Rahmenbedingungen in der Schweiz ${ }^{8}$}

Da die Angebote und Dienstleistungen in der Sozialwirtschaft mehrheitlich durch die öffentliche Hand finanziert werden, gilt es im Kontext von Versorgungsgestaltung, die politischen Rahmenbedingungen zu berücksichtigen. In Anlehnung an die Ausführungen von Paul Widmer (2008) bilden in der Schweiz die direkte Demokratie, der Föderalismus, die Neutralität sowie die sprachlich-kulturelle Vielfalt die vier Säulen des Schweizer Staatswesens und widerspiegeln die politische Kultur in der Schweiz (Widmer 2008: 10).

Im Kontext von Systemgestaltung in der Sozialwirtschaft sind insbesondere die ersten beiden Säulen des schweizerischen Staatswesens bedeutsam - die direkte Demokratie und der ausgeprägte Föderalismus.

\section{a) Direkte Demokratie}

Das politische System der Schweiz zeichnet sich durch eine konsequente Umsetzung des Prinzips der unmittelbaren Volkssouveränität aus. Im Vergleich zu parlamentarischen Demokratien entscheidet das Volk abschliessend über sämtliche Verfassungsfragen. Auch die Entscheide des Parlaments unterstehen dem Vorbehalt der Nachentscheidung durch die Schweizer Stimmbürger/-innen (Vatter 2014: 343). Im Rahmen dieses Systems findet ein enges Zusammenwirken zwischen der Exekutive, der Legislative und dem Souverän statt (ebd.). Das Entscheidungssystem basiert nach Wolf Linder und Sean Mueller (2012) auf folgenden verfassungsgestützten Kompetenzen des Volks, des Parlaments und der Regierung:

- $\quad$ «Das Volk, dem die höchste demokratische Legitimation zukommt, behält die Mitwirkung und Letztentscheidung in allen wichtigen Politikfragen, die auf 
Verfassungsstufe zu lösen sind. Dies wird gewährleistet durch die Volksinitiative und durch das obligatorische Referendum» (Linder/Mueller 2012: 265).

- «Das Parlament, dem die zweitgrösste Legitimation zukommt, entscheidet über die nächstwichtigen Fragen, und zwar auf Gesetzesstufe. In den meisten Fällen ist diese Entscheidung endgültig, aber sie steht unter dem Vorbehalt der Nachentscheidung durch das Volk: Eine Gruppe von Bürgern kann das fakultative Gesetzesreferendum ergreifen; in diesem Fall zieht das Volk die Letztentscheidung an sich» (ebd.).

- $\quad$ «Die Regierung, mit der geringeren demokratischen Legitimation als Volk und Parlament, ist für die Verordnungsgebung und Einzelentscheide geringerer Tragweite zuständig. Die Regierung handelt hier in einem eigenen Kompetenzbereich und unabhängig vom Parlament. Diese Unabhängigkeit gilt auch gegenüber dem Volk. Es gibt keine Nachkontrolle der Regierungsentscheidungen durch das Referendum, und im Prinzip auch keine Volksinitiative für Regierungsgeschäfte. Dies gilt sinngemäss auch für Parlamentsbeschlüsse von geringer Tragweite» (ebd.).

Das beschriebene demokratische Grundkonzept kann in der Schweiz auf Bundes-, Kantons- und Gemeindeebene gefunden werden (Linder/Mueller 2012: 268).

b) Ausgeprägter Föderalismus

Weiter ist das Schweizer Staatswesen durch einen ausgeprägten Föderalismus gekennzeichnet, durch den die Kompetenzen vertikal zwischen Bund, Kantonen und Gemeinden aufgeteilt sind (Widmer 2008: 103). Kernstücke des schweizerischen Bundesstaats sind die in der Bundesverfassung verankerte Autonomie der Kantone, deren Gleichberechtigung und Mitwirkung an der Willensbildung des Bunds sowie die Zusammenarbeitspflicht (Vatter 2014: 427).

Als kleinste Verwaltungseinheiten mit vielfältigen Zuständigkeiten nehmen die politischen Gemeinden als öffentlich-rechtliche Gebietskörperschaften eine wichtige Funktion ein und sind in einzelnen Politikfeldern mit bedeutenden Kompetenzen ausgestattet. Zentrale Merkmale der Gemeinden sind die Gemeindeautonomie, das Subsidiaritätsprinzip, die grosse Zahl an kleinen Kommunen in der Schweiz sowie die hohe Diversität der Gemeinden (Vatter 2014: 436ff.).

Die Autonomie der Kantone wird zum Beispiel in der 1999 revidierten Bundesverfassung im Artikel 3 deutlich: «Die Kantone sind souverän, soweit ihre Souveränität nicht durch die Bundesverfassung beschränkt ist; sie üben alle Rechte aus, die nicht dem Bund übertragen sind». In diesem Verfassungsartikel wird gleichzeitig das Subsidiaritätsprinzip sichtbar, das staatliche Aufgaben den Kantonen zuordnet, die nicht explizit in Bundeszuständigkeit fallen. Nach Jean- 
François Aubert (1978) und gemäss Dietmar Braun (2003) können die zentralen Prinzipien der kantonalen Autonomie wie folgt konkretisiert werden:

- Die Existenz der Kantone ist garantiert und ihnen steht es frei, wie sie sich im Inneren organisieren: So geben sich die Kantone selbst je eine eigene Verfassung, welche der Bundesverfassung untergeordnet ist. Sie wählen auch ihre Organe selbständig, wobei auf Bundesebene festgelegte Prinzipien wie demokratische Staatsform, Gemeindeautonomie, Rechtsstaatlichkeit und adäquate Vollzugsorganisation zu beachten sind.

- Die Kantone verfügen über eigene finanzielle Ressourcen: Sie haben das Recht, eigene Steuern zu erheben. Auch die Gemeinden haben die Kompetenz, Steuern zu erheben.

- Die Kantone unterliegen keiner politischen Kontrolle: Der Bund kann nicht ohne rechtliche Grundlage in die Ausgestaltung der kantonalen Politik eingreifen.

- $\quad$ Die Kantone beteiligen sich gleichberechtigt am Willensbildungsprozess auf Bundesebene.

- Die Kantone besitzen ausgedehnte Kompetenzen: Wer eine Aufgabe dem Bund zuordnen möchte, muss zuerst den Beweis erbringen, dass diese nicht den Kantonen zusteht. Zudem sind die Kantone in den meisten Fällen auch für den Vollzug der Bundespolitik zuständig.

Auch in sozialpolitischen Belangen, bei denen in wesentlichen Sachgebieten wie der Alters- und Hinterlassenenversicherung (AHV), der Invalidenversicherung (IV) oder der Arbeitslosenversicherung (ALV) die Gesetzgebungskompetenz beim Bund liegt, wird der Vollzug der Bundespolitik weitgehend den Kantonen anvertraut. Die Kantone erhalten so die Möglichkeit, die Umsetzung von Programmen teilweise autonom zu gestalten und bei deren Umsetzung auf ihre besonderen Rahmenbedingungen Rücksicht zu nehmen.

In dieser Form des Vollzugsföderalismus treffen die programmierenden und die umsetzenden Verwaltungsebenen aufeinander und es ist eine Vielzahl von Akteuren mit unterschiedlichen Interessen involviert. Diese Nahtstelle zwischen nationaler Gesetzgebungsebene, Bundesämtern und kantonalen Umsetzungsverantwortlichen hat in der Operationalisierung von sozialwirtschaftlichen Zielen eine grosse Bedeutung. Dabei werden Programme konkretisiert, die Finanzierung gesprochen oder Strukturen, Zuständigkeiten, Zielgruppen und Leistungsberechtigte festgelegt. 
c) Zunehmende Politikverflechtung

Politikverflechtung im politikwissenschaftlichen Sinn meint nach Fritz W. Scharpf (Scharpf/Reissert/Schnabel 1976) und Arthur Benz (Benz/Dose 2004) die organisatorisch aufgeteilte, aber materiell auf vielfältige Weise ineinandergreifende Planung, Finanzierung und Durchführung von Aufgaben durch den Bund, die Kantone und die Gemeinden. Uwe Serdült und Walter Schenkel (2006) beschreiben das politische System der Schweiz als stark verflochten. Der aus einem Staatenbund hervorgegangene Föderalismus nach schweizerischem Muster bewirke, dass ganze Politikbereiche vorwiegend auf kantonaler und kommunaler Stufe umgesetzt werden müssten. Eine klare Abgrenzung zwischen den Ebenen von Bund, Kantonen und Gemeinden sei mit dem Ausbau des Sozialstaats nach dem Zweiten Weltkrieg immer weniger möglich geworden. Die betreffenden Autoren stellen weiter fest, dass sich bundesstaatliche Beziehungen nicht mehr alleine mit Hilfe von formal-institutionellen Modellen beschreiben lassen. Der moderne Staat könne nicht mehr als kompakt auftretender Akteur verstanden werden. Er stehe nicht mehr deutlich über gesellschaftlichen und privatwirtschaftlichen Organisationen.

In jüngster Zeit sind komplexe Netzwerke mit wechselnden Akteurskonstellationen und Beziehungsmustern entstanden (u.a. Jegen 2003, Klöti/Hirschi/Serdült/Widmer 2005, Trechsel/Sciarini 1998, Serdült 2002). Darin hat sich nicht nur die Rolle der staatlichen Akteure gewandelt, sondern auch diejenige nichtstaatlicher. So sind beispielsweise an der Planung, Entscheidungsfindung und Durchführung von drogenpolitischen Massnahmen in der Schweiz Akteure der Strafverfolgung, der Medizin und der Sozialwirtschaft auf allen drei Staatsebenen beteiligt (Cattacin 1996, Kübler 2000).

Zusammenfassend lässt sich nach Serdült und Schenkel (2006) feststellen, dass immer mehr staatliche und nichtstaatliche Akteure sowie Organisationen am politischen Entscheidungsprozess teilhaben. Dabei sind staatliche Stellen aus Kapazitätsgründen zunehmend auf die Mitwirkung nichtstaatlicher Akteure angewiesen.

\section{Folgerungen für zentrale Handlungskompetenzen der Systemgestaltung}

Wie aus den obigen Ausführungen folgt, sind die soziale Sicherheit und das Feld der Sozialwirtschaft eng mit den komplexen, kleinräumigen und föderalistisch geprägten Strukturen des schweizerischen Staats und des politischen Systems verschränkt. Die Systemgestaltung ist innerhalb dieser Strukturen eingebettet und die Gestaltungsmöglichkeiten sind von den jeweiligen Rahmenbedingungen geprägt. 
Dabei ist ein hohes Mass an Politikverflechtung und die Mitwirkung nichtstaatlicher Akteure zu berücksichtigen.

Dieses Umfeld erfordert eine ausgeprägte Handlungskompetenz im Verständnis verschiedener Systeme und ihrer unterschiedlichen Logiken (verschiedene Staatsebenen, komplexe Zuständigkeiten unterschiedlichste Akteure mit je eigenen Interessen usw.). Bei der Entwicklung und Systemgestaltung sind demzufolge in ausgeprägter Weise Systemübersetzungsleistungen zu erbringen. Systemverständnis und Systemübersetzung können daher als eine zentrale Handlungskompetenz formuliert werden (siehe Abschnitt 3.4.2). Diese zentrale Handlungskompetenz kann auch mit Sensitivität und Empathie für unterschiedliche Systemrealitäten und -logiken sowie einer hohen Übersetzungs- und Vermittlungskompetenz in der Kommunikation zwischen den unterschiedlich ausgerichteten Systemen» umschrieben werden.

\subsubsection{Finanzielle Rahmenbedingungen}

Ohne Finanzierung ist eine Systemgestaltung in der Sozialwirtschaft nicht denkbar, wobei sozialwirtschaftliche Leistungen oftmals durch Organisationen, die dem Dritten Sektor (Non-Profit-Bereich) zugeordnet werden können, erbracht werden. Der öffentliche Sektor ist ebenfalls stark vertreten und auch Akteure, die zum marktwirtschaftlichen Sektor gehören, sind in diesem Feld anzutreffen. Damit wird deutlich, dass sich die Finanzierungsfragen je nach Form der Trägerschaft unterscheiden. Marktwirtschaftliche Unternehmen erzielen über den Verkauf von Produkten und Dienstleistungen Gewinne, der öffentliche Sektor finanziert sich in erster Linie über öffentliche Mittel. NPO können sich über eine Mischung aus Mitgliederbeiträgen, Spenden, Sponsoring und Legaten und der Abgeltung von Leistungen finanzieren, die im Auftrag der öffentlichen Hand erbracht werden. Dabei kann vielfach eine Kombination dieser unterschiedlichen Handlungslogiken in einer Organisation angetroffen werden. Klaus Grunwald und Paul-Stefan Ross (zit. in Tabatt-Hirschfeldt 2014: 46) sprechen von einem Hybridisierungsphänomen.

Trotz dieser zunehmenden Überlagerung von Organisationsformen werden sozialwirtschaftliche Leistungen zum überwiegenden Teil aus öffentlichen Mitteln finanziert. Muss die öffentliche Hand sparen, schlägt sich dies auch in der Verknappung der Mittel für die Sozialwirtschaft nieder. 
Schönberger (2013, zit. in Ladner/Chappelet/Emery/Knoepfel/Mader/Soquel/Varone 2013: 566) beschreibt gemäss dem europäischen System der volkswirtschaftlichen Gesamtrechnung die Inhalte öffentlicher Haushalte. Demzufolge beinhalten öffentliche Haushalte Einheiten,

- $\quad$ die Steuern einnehmen und die durch Steuern und andere öffentliche Einnahmen finanziert werden oder

- $\quad$ Einkommen und Vermögen umverteilen und

- $\quad$ die unter staatlicher Kontrolle stehen (z.B. durch die Ernennung der Direktion), wobei nicht mehr als die Hälfte der Produktionskosten durch Einnahmen gedeckt sind, die über den Markt erwirtschaftet werden (Verkauf von Gütern, Dienstleistungen oder sonstige Leistungen).

Wie der zitierte Autor weiter ausführt, besteht in der Schweiz die öffentliche Hand aus vier institutionellen Einheiten, nämlich dem Bund, den 26 Kantonen und ihren verschiedenen Konkordaten, den Gemeinden und den obligatorischen öffentlichen Sozialversicherungen.

Die Sozialversicherungen finanzieren sich überwiegend über Sozialversicherungsbeiträge. Das Gros der öffentlichen Leistungen wird durch Steuereinnahmen und Gebühren finanziert. Allerdings erheben auch hier Bund, Kantone und Gemeinden je eigene Steuern. Die Höhe dieser Steuern wird durch direktdemokratische Prozesse mitbestimmt. So gab es zum Beispiel am 27. September 2009 eine eidgenössische Volksabstimmung für eine Zusatzfinanzierung der IV durch eine befristete Erhöhung der Mehrwertsteuer (2011-2017). Die Vorlage wurde angenommen und damit konnte die IV ihre finanzielle Situation verbessern. Diese Koppelung von direkter Demokratie und Finanzierung führt dazu, dass die öffentlichen Ausgaben ständig einem hohen Legitimationsdruck ausgesetzt sind.

Kantone und Gemeinden legen die Höhe ihrer Steuern unter Berücksichtigung von rechtlichen Rahmenbedingungen selber fest. Dies führt zu einem ständigen Steuerwettbewerb unter den Kantonen und den Gemeinden, wobei auf nationaler Ebene ein Finanzausgleich für eine gewisse Begrenzung der Unterschiede zwischen finanzstarken und finanzschwachen Kantonen führt. Entsprechende Ausgleichsmechanismen gibt es innerhalb der Kantone in Bezug auf die Gemeinden.

Die komplexen sozialpolitischen Rahmenbedingungen sind mit entsprechend komplexen Finanzierungsmodellen verknüpft, die bei der Systemgestaltung in der Sozialwirtschaft zu berücksichtigen sind. Die Kosten sind gegenüber den Stimmbürgerinnen und Stimmbürgern immer wieder zu legitimieren, da mit direktdemokratischen Instrumenten direkt auf die Finanzen der öffentlichen Hand Einfluss genommen werden kann. 


\section{Folgerungen für zentrale Handlungskompetenzen der Systemgestaltung}

Bei der Systemgestaltung ist daher ständig im Auge zu behalten, wie die Leistungen von den Bürgerinnen und Bürgern wahrgenommen und wie Erstere im öffentlichen, medialen und politischen Diskurs erörtert werden. Die Sozialwirtschaft muss ihren Mehrwert und Nutzen, den sie für die Gesellschaft erbringt, öffentlich sichtbar machen und aktiv kommunizieren. Andernfalls werden die benötigten finanziellen Ressourcen nicht zur Verfügung gestellt. In der direkten Demokratie der Schweiz ist diesem Kontext der Legitimation eine besondere Bedeutung beizumessen. Daher stellt eine adressatengerechte Kommunikation und Erläuterung sozialer Angebote und Dienstleistungen eine zentrale Handlungskompetenz dar. Die Basis dieser Kommunikationsleistungen stellen Systemverständnis- und Systemübersetzungskompetenzen sowie Sozialwirtschaftskompetenzen dar.

\subsection{3 Ökonomisierung der Sozialen Arbeit}

Obwohl in den letzten Jahren viel über die Ökonomisierung der Sozialen Arbeit geschrieben wurde, ist von dieser keine einheitliche Definition in den einschlägigen Nachschlagewerken zu finden (Tabatt-Hirschfeldt 2014). Es erscheinen fortwährend die gleichen oder ähnliche Schlüsselwörter, wenn über die Ökonomisierung gesprochen wird: Wirtschaftlichkeit und Sparsamkeit bei der Leistungserbringung, Effizienz und Effektivität, Wettbewerb, Neoliberalisierung, Privatisierung, Kostenvergleich und Benchmark, Wirkungsmessung und so weiter.

Es ist in diesem Kontext nicht zu übersehen, dass diese verschärfte Ökonomisierung nicht nur die Marktorientierung von sozialen Diensten und Einrichtungen forciert, sondern auch die innere Identität, das heisst das Selbstverständnis der Organisationen nachhaltig beeinflusst. Die Verunsicherung ist teilweise gross, die Suche nach Orientierung und neuen Leitideen ebenso. Das zeigt sich auch in den vielen Entwicklungsprojekten, die in sozialen Institutionen am Laufen sind: Es gibt wohl kaum eine Organisation, die in den letzten Jahren nicht ein Leitbild erarbeitet oder sich mit der Neupositionierung, mit Fusionen und Kooperationen und / oder mit einem neuen «Branding» auseinandergesetzt hat.

Es existiert jedoch nicht nur die «Ökonomisierung nach innen» - in die eigene Organisation -, sondern auch die «Ökonomisierung nach aussen» - in das Versorgungssystem. Durch die Einführung wettbewerblicher Elemente zwischen den Leistungserbringenden, durch einen deutlich erhöhten Legitimationsnachweis gegenüber den Leistungsbestellenden und -finanzierenden, durch das Aufkommen privater, gewinnorientierter Anbietenden von sozialen Dienstleistungen sowie 
durch eine zunehmend formalisierte und oft auch bürokratisierte Beziehung zwischen «Anbietenden und Bestellenden» sind neue Prämissen gesetzt worden.

Es können drei grundsätzliche Reaktionen auf die vielfältigen Formen der Ökonomisierung beobachtet werden (Tabatt-Hirschfeldt 2014, Sommerfeld/Haller 2003):

(1) Abwehr gegen alles Ökonomische: Es bestehen - vermutlich immer weniger - soziale Organisationen, die sich grundsätzlich ökonomischen Anforderungen verschliessen, weil diese Grundwerte der Sozialen Arbeit tangieren: Helfen sei Dienst am Nächsten und lasse sich nicht in Kategorien der Betriebswirtschaftslehre fassen. NPO seien besondere Institutionen, die nicht mit gewinnorientierten verglichen werden könnten. Die Ökonomie und der Markt zeigten unmenschliche Züge.

(2) Übernahme alles Neuen: Bei diesem Reaktionsmuster findet eine blinde Übernahme aller neuer Management- und Betriebswirtschaftskonzepte statt. Begriffe werden unreflektiert übernommen: Klientinnen und Klienten werden zu Kundinnen und Kunden, soziale Organisationen zu Unternehmungen. Es wird von Markt gesprochen - was die Möglichkeit der Wahl beinhaltet, wo es diese Wahl gar nicht gibt (z.B. in der gesetzlichen Sozialarbeit). Das «doppelte Mandat» (Böhnisch/Lösch 1973) oder das «dreifache Mandat» (Staub-Bernasconi 2007) verschwinden, weil nur noch ein Auftrag der Leistungsfinanzierenden existiert und keine Klientinnen und Klienten.

(3) Auseinandersetzung mit der Ökonomie: Soziale Dienstleistungen definieren sich primär durch eine fachlich kompetente und ethisch begründbare Arbeit. Zudem - aber nicht primär - müssen sie sich auch mit den betriebswirtschaftlichen und anderen ökonomischen Ansprüchen auseinandersetzen. Soziale Organisationen sind aus einem sozialpolitischen Auftrag heraus entstanden und deshalb ist es nach dieser Auffassung auch wichtig, dass sie das, was sie tun, auch begründen und legitimieren können. Deshalb darf sich die Soziale Arbeit ökonomischen Konzepten nicht verschliessen; sie muss diese jedoch, zum Beispiel beim Qualitätsmanagement, fachlich mitdefinieren. Diese Aufgabe ist anspruchsvoll und per se auch von Widersprüchen geprägt - dies ist geradezu die Kunst in der Führung einer sozialen Organisation. 


\section{Folgerungen für zentrale Handlungskompetenzen der Systemgestaltung}

Es dürfte deutlich geworden sein, dass sich die Autoren des vorliegenden Handbuchs am dritten Verständnis orientieren, das für eine Auseinandersetzung von Ökonomie in der Sozialen Arbeit plädiert. Für die Systemgestaltung wird demzufolge neben der fundierten Fachkompetenz in der Sozialen Arbeit und einschlägiger Branchenkenntnis ebenso ein betriebswirtschaftliches Handwerk verlangt, das heisst eine Verbindung von Fachkompetenz im Feld der Sozialen Arbeit und ökonomischer Kompetenz. Die Autoren sprechen in diesem Zusammenhang von Sozialwirtschaftskompetenz (siehe Abschnitt 3.4.1).

\subsubsection{Welfare-Mix}

Die Organisationen der Sozialwirtschaft sind heute einem vielfältigen Spannungsfeld ausgesetzt, wie bisher in diesem Kapitel aufgezeigt wurde: den sozialpolitischen und finanziellen Rahmenbedingungen der öffentlichen Hand, einer starken Ökonomisierung der Managementaufgaben und einer zunehmenden Wettbewerbsorientierung in der Sozialwirtschaft zwischen wirtschaftlicher Rationalität und fachlich-professioneller Qualität. Diese Entwicklungen führen auch zu einem Wandel im Professionsverständnis der Sozialen Arbeit.

Es wurde oben darauf hingewiesen, dass die in der Sozialwirtschaft ausgeführten Leistungen durch mehr und teilweise neue Akteure angeboten werden: durch die öffentliche Hand (Staat), durch NPO, durch profitorientierte Unternehmen wie auch durch primäre, informelle Netzwerke wie Nachbarschaften oder soziale Bewegungen, die aus einem zivilgesellschaftlichen Engagement entstehen. Angesichts dieser Angebotsvielfalt ist die Komplexität der Angebotsstrukturen sehr vielfältig und heterogen geworden, so dass in dieser Hinsicht von einer «neuen Unübersichtlichkeit» im «Wohlfahrtsmix» (Grunwald/Ross 2014) gesprochen werden kann.

Der Begriff des Welfare-Mix, Wohlfahrtsmix oder Wohlfahrtspluralismus wird seit den 1990er Jahren in Deutschland verwendet (Evers/Olk 1996) und wurde als analytisches Konzept in den Jahren 2011 und 2012 von Adalbert Evers und Paul-Stefan Ross weiterentwickelt. Evers und Ross gehen davon aus, dass in modernen, ausdifferenzierten Gesellschaften unterschiedliche, untereinander konkurrierende Ordnungsprinzipien in unterschiedlichen Mischungsverhältnissen an der Erbringung von Wohlfahrt beteiligt sind. Dies geschieht zudem in vier verschiedenen gesellschaftlichen Sektoren: dem informellen Sektor, dem Assoziativen (Dritten) Sektor, dem Staat sowie dem Markt. Diesen Sektoren lassen sich je 
eigene Systemlogiken, Zugangsregeln und Zentralwerte zuordnen, wie sie in der untenstehenden Abbildung aufgezeigt sind.

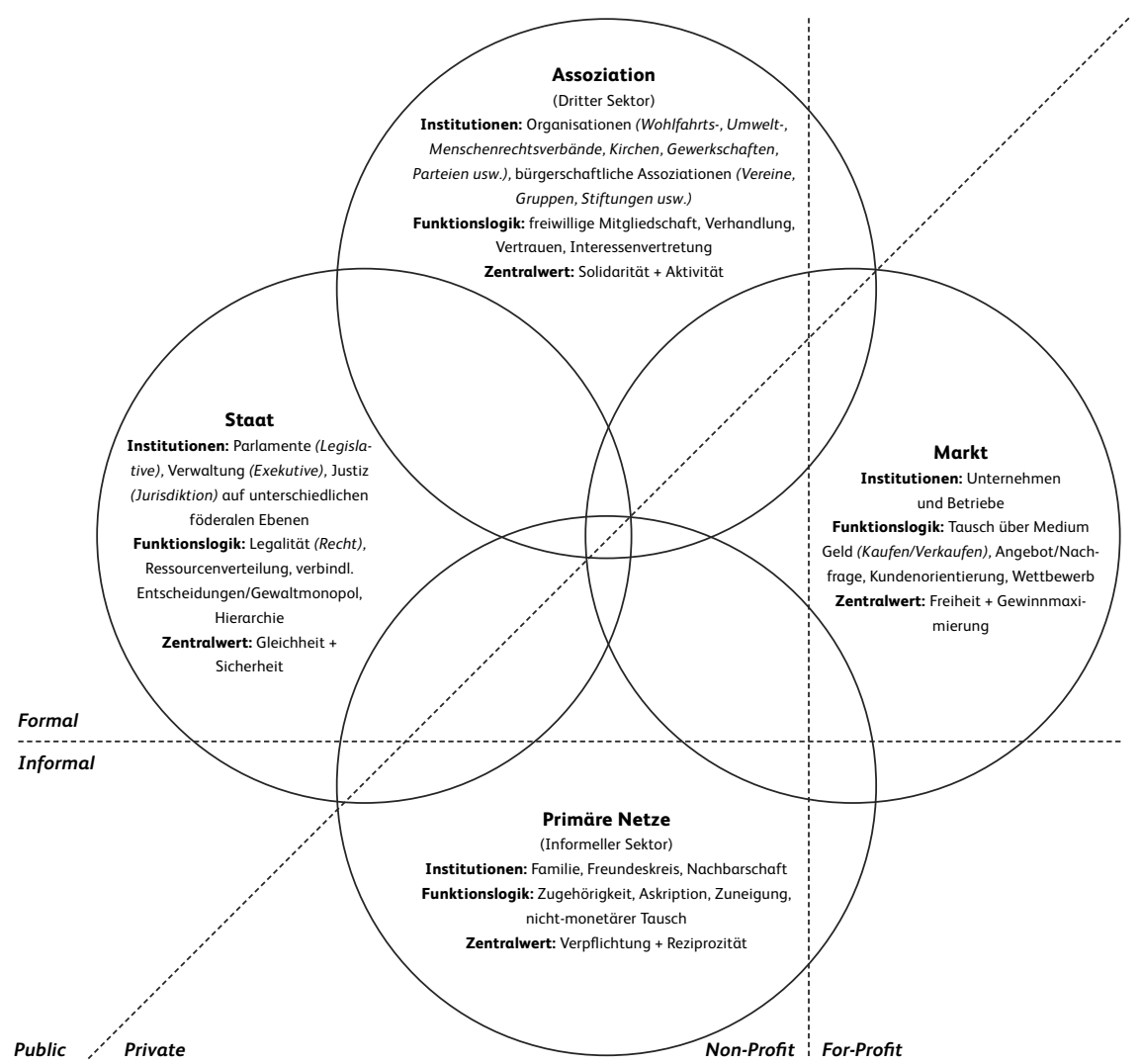

Abbildung 8: Sektoren gemischter Wohlfahrtsproduktion

Quelle: Ross (2012: 317)

Ross (2012) weist darauf hin, dass die genannten vier Sektoren nicht homogen sind und sich auch nicht trennscharf voneinander abgrenzen lassen. Jeder Sektor verfügt hingegen über eine bestimmte Funktionslogik und über Zentralwerte, wie sie im obigen Schaubild aufgeführt sind. 
Für die Systemgestaltung relevant sind die spezifischen Systemstärken und Systemschwächen im Hinblick auf die Erbringung sozialer Leistungen (siehe $A b$ bildung 9).

\begin{tabular}{|c|c|c|}
\hline & Spezifische Systemstärken & Spezifische Systemschwächen \\
\hline Primäre Netze & $\begin{array}{l}\text { Emotionale Nähe } \\
\text { Direkte, wechselseitige Unter- } \\
\text { stützung im Alltag }\end{array}$ & $\begin{array}{l}\text { Überforderung / mangelnde } \\
\text { Verlässlichkeit } \\
\text { Bevormundung und soziale } \\
\text { Verstrickungen }\end{array}$ \\
\hline $\begin{array}{l}\text { Assoziationen } \\
\text { (Dritter Sek- } \\
\text { tor) }\end{array}$ & $\begin{array}{l}\text { Freiwilligkeit von Ein- und Austritt } \\
\text { Abbildung und Bündelung pluraler } \\
\text { Interessen / Werte, freie } \\
\text { Meinungsbildung } \\
\text { Identifizierung von Handlungs- } \\
\text { bedarfen, Umsetzung in Aktion } \\
\text { Ermöglichung persönlicher } \\
\text { Solidarität }\end{array}$ & $\begin{array}{l}\text { Voraussetzungsreich hinsichtlich } \\
\text { Entstehung und Bestand } \\
\text { Hohe Heterogenität } \\
\text { Eingeschränkte Durchsetzungsmacht } \\
\text { Aufwändige Ressourcengewinnung }\end{array}$ \\
\hline Staat & $\begin{array}{l}\text { Durchsetzung verbindlicher } \\
\text { Regelungen } \\
\text { Gewährleistung von Sicherheit, } \\
\text { Legalität und Rechtsansprüchen } \\
\text { Umverteilung von Ressourcen } \\
\text { entsprechend legitimierter } \\
\text { Prioritätensetzungen }\end{array}$ & $\begin{array}{l}\text { Langsame Reaktion auf Verände- } \\
\text { rungen / Innovationsanforderun- } \\
\text { gen } \\
\text { Tendenz zu Bürokratie / } \\
\text { Abkoppelung } \\
\text { Angewiesenheit auf politische } \\
\text { Mehrheiten }\end{array}$ \\
\hline Markt & $\begin{array}{l}\text { Versorgung mit nachgefragten } \\
\text { Gütern } \\
\text { Wertschöpfung } \\
\text { Innovationskraft, Anpassungsfä- } \\
\text { higkeit an veränderte Bedarfe } \\
\text { Produktionsverbesserung und / } \\
\text { oder Kostensenkung durch } \\
\text { Wettbewerb }\end{array}$ & $\begin{array}{l}\text { Abhängigkeit von zahlungskräfti- } \\
\text { ger Nachfrage, Verschwinden } \\
\text { wenig nachgefragter Produkte } \\
\text { Ungleichverteilung von Gütern } \\
\text { Einebnung spezifischer Werte }\end{array}$ \\
\hline
\end{tabular}

Abbildung 9: Spezifische Systemstärken und -schwächen der Sektoren Quelle: Ross (2012: 319)

Da die Funktionslogiken und Werte dieser vier Sektoren unterschiedlich sind, stellt sich die Frage nach Dominanz, Einfluss und Macht der einzelnen Sektoren. Hier müssten wohl verschiedene Ebenen der Ausbalancierung betrachtet werden. Im globalen Sinn auf der Makroebene geht es um die Frage, welche Form von sozialstaatlicher Politik und Steuerung sich durchsetzt, auf der Mesoebene um die Vielfalt und Hybridisierung unterschiedlicher organisationaler Logiken und auf der Mikroebene um die personalisierte Einflussnahme über die Sektoren hinweg. 
Grunwald und Ross (2014: 24) erwähnen fünf zentrale Trends, die sich seit einiger Zeit in diesem Wohlfahrtsmix erkennen lassen:

- $\quad$ Der informelle Sektor ${ }^{9}$ wird zunehmend mehr und systematisch einbezogen.

- $\quad$ Es werden verstärkt privat-gewerbliche Anbietende einbezogen; die Landschaft der Anbietenden sozialer Dienstleistungen diversifiziert sich.

- Der Staat wird zunehmend in der Rolle als Initiator, Regulator und Moderator gesehen. Ihm wird eine (überwiegend durch Kontextsteuerung wahrzunehmende) Gewährleistungsfunktion für Wohlfahrt zugewiesen.

- Individualisierte und «personalisierte Versorgungsgestaltung» (Wendt 2010: 10) im Sinn massgeschneiderter personen- und situationsbezogener Hilfen gewinnt an Bedeutung.

- $\quad$ Es wird systematisch versucht, freiwilliges bürgerschaftliches Engagement als festen Bestandteil in die Dienstleistungserbringung einzubeziehen.

Diese Trends beziehen sich auf Deutschland, dürften aber weitgehend auch für die Schweiz gelten. Dabei ist zu berücksichtigen, dass diese Entwicklungen nicht einfach auf die Schweiz mit ihren vielen kleinen und mittelgrossen Einrichtungen, einem ausgeprägten Föderalismus und der direkten Demokratie in kleineren geografischen, sozialen und verwaltungstechnischen Räumen übertragen werden können.

\section{Folgerungen für zentrale Handlungskompetenzen der Systemgestaltung}

Die Systemgestaltung im System einer gemischten Wohlfahrtsproduktion ist von einer beträchtlichen Anzahl und Vielfalt unterschiedlicher Akteure geprägt. Dies stellt zunehmende Anforderungen an die Zusammenarbeit und Kooperationen zwischen diesen. Daraus leitet sich eine weitere zentrale Handlungskompetenz für die Systemgestaltung ab: die Vernetzungs- und Kooperationskompetenz (siehe $A b$ schnitt 3.4.4). Dabei geht es darum, die Sachverständigkeit und das Handwerkszeug verschiedenster Akteure mit ihren unterschiedlichsten Anliegen, Interessen und Leistungskapazitäten in eine produktive und verbindliche Form der Zusammenarbeit zu bringen.

9 Der informelle Sektor umfasst alle ökonomischen Aktivitäten, die sich ausserhalb der formell geregelten Strukturen bewegen (Sellien 2004: 1486). 


\subsubsection{Differenz von Sozial- und Verwaltungsraum}

Der Begriff des Sozialraums ist für die Systemgestaltung wesentlich, da sozialpolitische Steuerung immer innerhalb eines mehr oder weniger klar definierten geografischen Raums stattfindet.

In Theorie und Praxis finden sich sehr unterschiedliche Bedeutungen des Begriffs «Sozialraum». Andreas Hopmann (zit. in Schönig 2008: 13) unterscheidet zwischen:

- Sozialraum als Territorium (Verwaltungsperspektive);

- Sozialraum als gebündelte Lebenswelt (Bewohnerperspektive);

- $\quad$ Sozialraum als Ziel (Steuerungsgrösse);

- $\quad$ Sozialraum als Ressource (Mittel);

- $\quad$ Sozialraum als Einzugsbereich (Marktgebiet einer Einrichtung);

- $\quad$ Sozialraum als Ort der Sozialen Arbeit (öffentlicher Raum oder Gebäude).

Diese Vielfalt nimmt auch die Umschreibung des «Sozialraums» von Mathias Schwabe (2015: 1) auf:

«Sozialraum meint einen Lebensraum von Menschen (...), der durch eine bestimmte geografische Ausdehnung definiert und aus einer spezifischen Perspektive heraus wahrgenommen wird. Von der Ausdehnung her kann ein Sozialraum eine ganze Stadt [oder] zwei angrenzende Bezirke ebenso umfassen wie eine Siedlung, einen Strassenzug oder zwei benachbarte Hochhäuser, kann bestimmter Sozialraum ebenso auf dem Land (....) wie in einer Grossstadt gelegen sein. Von der Betrachtungsperspektive her kann ein Sozialraum ebenso ein nach Verwaltungskriterien vorgegebenes Gebiet darstellen (Bezirk, statistisches Gebiet, (....) wie einen nach subjektiven Kriterien emotional empfundenen sozio-kulturellen Zusammenhang (Kiez, Veddel, Siedlung,...). Im offiziellen Sozialraum der Verwaltung existieren also noch [T] ausende Sozialräume von Bürgerinnen und Bürgern, Kindern, Jugendlichen etc. Sozialräume können als 'neutrale Gebilde' oder als 'eigen', 'behaglich', 'Heimat' betrachtet werden, aber auch als 'Wespennest', 'Kriegsschauplatz', 'ödes Kaff' erlebt werden. Ein und derselbe Sozialraum (....) kann von unterschiedlichen Nutzerinnen und Nutzern mit unterschiedlichen Zielen aufgesucht werden, was zu erheblichen Interessenkonflikten führen kann».

Der sozialraumorientierten Gestaltung in der Sozialwirtschaft kann ein zweistufiger Raumbegriff zugrunde gelegt werden (Schönig 2008: 17): einerseits die Perspektive der Sozialverwaltung, die die Grenzen des Sozialraums politisch-administrativ zieht, und andererseits die Perspektive der jeweiligen Zielgruppe, für die - je nach Handlung und Sinnzuschreibung - davon abweichende Grenzen des Sozialraums relevant sind. 
Aus der Sicht der Sozialplanung wird im Handbuch Sozialplanung des Bundeslands Nordrhein-Westfalen empfohlen, auf Basis statistischer Grundlagen und unter Berücksichtigung konkreter örtlicher Verhältnisse Sozialräume als einheitliche Bezugsbasis für sämtliche Planungsprozesse zu definieren (Reichwein/Berg/Glasen/Junker/Rottler-Nourbakhsch/Vogel 2011: 40). Hierbei wird im Weiteren auf eine quartier- oder stadtteilbezogenen Perspektive verwiesen. Damit wird eine Brücke zu einer integrierten Stadt- und Regionalplanung geschlagen. Dieses Verständnis einer sozialraumorientierten Planung gilt es mit der stark föderalistisch ausgeprägten Struktur in der Schweiz und mit der dadurch gegebenen komplexen Organisation der sozialen Sicherungssysteme zu verbinden. Am Beispiel der Strukturen für Personen im erwerbsfähigen Alter soll deutlich gemacht werden, wie komplex die sozialen Sicherungssysteme in der Schweiz organisiert sind ( $A b$ bildung 10). 


\begin{tabular}{|c|c|c|c|}
\hline $\begin{array}{l}\text { Staatliche } \\
\text { Ebene }\end{array}$ & $\mathbf{A L V}$ & IV & Sozialhilfe \\
\hline Bund & $\begin{array}{l}\text { Bundesbehörden } \\
\text { Staatssekretariat für } \\
\text { Wirtschaft, Direktion } \\
\text { für Arbeit }\end{array}$ & $\begin{array}{l}\text { Bundesbehörden } \\
\text { Bundesamt für Sozi- } \\
\text { alversicherungen, } \\
\text { Geschäftsfeld IV }\end{array}$ & \\
\hline $\begin{array}{l}\text { Interkanto- } \\
\text { nale Koor- } \\
\text { dination }\end{array}$ & $\begin{array}{l}\text { Konferenz kantonaler } \\
\text { Volkswirtschaftsdi- } \\
\text { rektoren } \\
\text { Verband Schweizeri- } \\
\text { scher Arbeitsmarktbe- } \\
\text { hörden }\end{array}$ & $\begin{array}{l}\text { IV-Stellen- } \\
\text { Konferenz }\end{array}$ & $\begin{array}{l}\text { Konferenz der kantona- } \\
\text { len Sozialdirektorin- } \\
\text { nen und Sozialdirekto- } \\
\text { ren } \\
\text { Schweizerische Konfe- } \\
\text { renz für Sozialhilfe }\end{array}$ \\
\hline $\begin{array}{l}\text { Kantons- } \\
\text { übergrei- } \\
\text { fend }\end{array}$ & & $\begin{array}{l}\text { Regionale ärztliche } \\
\text { Dienste }\end{array}$ & \\
\hline Kantone & $\begin{array}{l}\text { Kantonales Arbeitsamt } \\
\text { Kantonale Arbeitslo- } \\
\text { senkasse }\end{array}$ & $\begin{array}{l}\text { Kantonale IV-Stelle } \\
\text { Kantonale Sozial- } \\
\text { versicherungsan- } \\
\text { stalt } \\
\text { Kantonale Aus- } \\
\text { gleichskassen der } \\
\text { AHV / IV }\end{array}$ & $\begin{array}{l}\text { Kantonales Sozialamt } \\
\text { (KSA) }\end{array}$ \\
\hline Kantonal & $\begin{array}{l}\text { Regionale Arbeitsver- } \\
\text { mittlungszentren }\end{array}$ & & $\begin{array}{l}\text { Regionaler Sozialdienst } \\
\text { oder kommunales So- } \\
\text { zialamt }\end{array}$ \\
\hline Kommunen & & & Sozialkommission \\
\hline $\begin{array}{l}\text { Andere } \\
\text { Akteure }\end{array}$ & $\begin{array}{l}\text { Anbietende von Wie- } \\
\text { dereingliederungs- } \\
\text { massnahmen } \\
\text { Arbeitslosenkassen }\end{array}$ & $\begin{array}{l}\text { Anbietende von } \\
\text { Wiedereingliede- } \\
\text { rungsmassnahmen } \\
\text { Ausgleichskassen } \\
\text { Berufsvorsorgeeinrich- } \\
\text { tungen }\end{array}$ & $\begin{array}{l}\text { Anbietende von Wie- } \\
\text { dereingliederungs- } \\
\text { massnahmen }\end{array}$ \\
\hline
\end{tabular}

Abbildung 10: Aufgaben und Zuständigkeiten der sozialen Sicherung Quelle: Champion/Bonoli (2013), zitiert in Ladner/Chappelet/Emery/ Knoepfel/Mader/Soquel/Varone (2013: 708)

Es zeigt sich, dass bei der Sozialhilfe die Bundesebene nicht involviert ist und eine regionale, kantonsübergreifende Ebene nur bei den regionalärztlichen Diensten der IV zum Tragen kommt. Cyrielle Champion und Giuliano Bonoli weisen darauf hin, dass obige Darstellung stark vereinfachend ist, da einige Akteure wie die Unfall- oder Krankenversicherungen weggelassen wurden, die in bestimmten Gebieten eine wichtige Rolle spielen. Das System der sozialen Sicherheit für Personen im erwerbsfähigen Alter schliesst staatliche und private Akteure mit ein und trotz dieser Komplexität ist eine Zusammenarbeit unter den verschiedenen Akteuren 
notwendig, damit die involvierten Instanzen den gesetzlichen Auftrag umsetzen können.

Eine sozialraumorientierte Systemgestaltung hat - je nach Ebene und Perspektive der gestaltenden Akteure - eine unterschiedliche Ausrichtung. Kantonale Verwaltungsstellen orientieren sich in der Planung am Territorium des jeweiligen Kantons, wobei die Kantone je nach Grösse und geografischen Gegebenheiten oft in Planungsregionen aufgeteilt sind. Komplizierend kommt dazu, dass die einzelnen Institutionen wie die IV, die ALV oder die Sozialhilfe strukturell unterschiedlich und weitgehend unabhängig voneinander entwickelt und aufgebaut worden sind. So sind die regionalen Arbeitsvermittlungszentren in den einzelnen Kantonen unterschiedlich organisiert. Die IV-Stellen verfügen über eine kantonale Anlaufstelle und die Sozialhilfe ist je nach Kanton kommunal, regional, kantonal oder in Mischformen dieser Zuständigkeiten organisiert.

Für Anbietende sozialer Dienstleistungen wie zum Beispiel im Fall von Arbeitsintegrationsprogrammen sieht eine sozialraumorientierte Gestaltung wieder anders aus. Möglicherweise arbeitet eine Trägerschaft mit allen drei oben beschriebenen sozialen Sicherungssystemen zusammen und das Angebot wird über die Kantonsgrenzen hinaus angeboten.

Diese komplexe Organisation der sozialen Sicherheit schränkt eine idealtypische sozialraumorientierte Gestaltung ein. Massgebend ist die gesetzlich verankerte und tradierte Orientierung an Gemeinden, Kantonen und der Bundesebene. Insbesondere im Kontext der Sozialwirtschaft in der Schweiz werden diese politisch definierten Sozialräume noch wenig durchbrochen; dies im Gegensatz zu anderen Planungsanliegen wie dem Verkehr oder der Energieversorgung. Für die konkrete Gestaltung und Praxis sind daher die jeweiligen lokalen Rahmenbedingungen entscheidend.

\section{Folgerungen für zentrale Handlungskompetenzen der Systemgestaltung}

Im Rahmen der Systemgestaltung gilt es zu klären, um welche Art von Sozialraum es sich handelt und welche Kriterien die Grenzziehung eines Versorgungssystems bestimmen. Dabei ist zu berücksichtigen, dass zum einen der Gesichtspunkt der politisch-administrativen Zuständigkeiten besteht und zum anderen der Blickwinkel der Zielgruppen, welche die Grenze «ihres» Sozialraums aufgrund von Zugehörigkeit oder alltäglichen Lebensbezügen definieren. Die Abstimmung und die fachliche Integration dieser Perspektiven erfordern ein hohes Mass an Konzeptualisierungs- und Entwicklungskompetenz. Diese Handlungskompetenz umfasst vorausschauendes, strategisches und innovatives, zukunftsorientiertes Denken sowie analytische Fähigkeiten, um entsprechende Fachkonzepte zur Systemgestaltung entwickeln und verfassen zu können. 


\subsection{Theoriebezüge ausgewählter Handlungskonzepte}

Das Handeln von Fachleuten wird wesentlich von Erfahrungen und Werten sowie von Theorien und Handlungskonzepten bestimmt. Mit professionellem Handeln ist der Anspruch an die Fachpersonen verbunden, das eigene Tun zu begründen und dabei auf bestimmte Handlungskonzepte und Theorien Bezug zu nehmen. Im untenstehenden Abschnitt wird zuerst Bezug auf die Theorieentwicklung im Bereich Sozialmanagement und Sozialwirtschaft genommen, worauf in den folgenden Abschnitten Konzepte und Modelle, die den Buchautoren für eine professionelle Systemgestaltung wichtig erscheinen, kurz erläutert werden. Die Autoren versuchen dazu jeweils Bezug zu nehmen auf die eingangs erwähnten und im $A b$ schnitt 3.4 näher erläuterten Kernkompetenzen.

\subsubsection{Theorieentwicklung zu Sozialmanagement und Sozialwirtschaft}

Seit den 1990er Jahren hat sich die fachliche und wissenschaftliche Bearbeitung des Fachbereichs Sozialmanagement, oft auch «Management in der Sozialen Arbeit» genannt, sprunghaft ausgebreitet. Lange Zeit war dabei das Verhältnis zwischen Sozialer Arbeit und Management konfliktgeladen, da es von gegenseitigen Vorurteilen geprägt war. Die bereits erwähnte Ökonomisierung hat diesbezüglich zu einer breiten Fachdiskussion geführt. Die Zahl der Weiterbildungs- und Masterangebote zum Thema Sozialmanagement hat in kurzer Zeit enorm zugenommen. Karl-Heinz Boessenecker und Andreas Markert haben 2007 in ihrer Untersuchung im deutschsprachigen Raum bereits über 100 Studiengänge mit etwa 2'000 Absolventinnen und Absolventen erfasst (siehe Boessenecker/Markert 2011). Die Internationale Arbeitsgemeinschaft Sozialmanagement / Sozialwirtschaft (INAS) hat sich der theoretischen Vertiefung dieses Themenbereichs angenommen. Die Schweiz hat sich 2008 in Luzern mit der Durchführung der Tagung «Sozialwirtschaft und Sozialmanagement im deutschsprachigen Raum, Bestandsaufnahme und Perspektiven» erstmals offiziell an diesem Diskurs beteiligt (Bassarak/Wöhrle 2008). Daraus hervorgegangen ist nicht nur die Neubelebung der INAS, sondern in der Schweiz auch die Fachkommission Sozialmanagement, eine Untergruppe der Schweizerischen Gesellschaft für Soziale Arbeit. An den Schweizer Fachhochschulen sind inzwischen mehrere Publikationen (z.B. Fritze/Uebelhart 2015, Wüthrich/Amstutz/Fritze 2015) veröffentlicht worden. In dieser Tradition wird auch das vorliegende Buch verstanden; es schliesst an den Beitrag «Skizze eines systemischen Managementmodells für den Sozialbereich» (Bürgisser/Buerkli/Stremlow/Kessler/Benz 2012) an, der im dreibändigen Werk «Auf der Suche nach Sozialmanagementkonzepten für und in der Sozialwirtschaft» (Wöhrle 
2012: 231ff.) veröffentlicht wurde. Im Buch «Grundlagen des Managements in der Sozialwirtschaft» (Wöhrle/Beck/Grunwald/Schellberg/Schwarz/Wendt 2013) lässt sich der jüngere Diskussionsstand nachlesen.

In den letzten Jahren hat sich der Fokus der Diskussion vom Management einer einzelnen Organisation auf die Steuerung von Versorgungssystemen in der Sozialwirtschaft ausgeweitet. Es gibt inzwischen unzählige Konzepte und Lehrbücher darüber, wie eine soziale Organisation geleitet beziehungsweise geführt werden soll. Deutlich weniger Grundlagen existieren jedoch zur Frage, wie die vielfältigen Angebote in einem bestimmten Fachbereich, zum Beispiel dem Alters- oder Behindertenbereich, besser aufeinander abgestimmt und gezielt weiterentwickelt - eben gesteuert oder gestaltet - werden sollen. Die Autoren des vorliegenden Buches stellen auch eine zunehmende Nachfrage nach solchen Projekten fest, die von Fachpersonen im Bereich der Sozialplanung an sie herangetragen werden. Dies hat sie auch dazu bewogen, sich vertiefter mit den theoretischen Bezügen und methodischen Konzepten zur Systemgestaltung auseinanderzusetzen. Ausgehend von einem systemtheoretischen Ansatz nach Luhmann, wie er im Abschnitt 2.1 vorgestellt wurde, haben die Autoren Theoriebezüge aus der systemischen Organisationslehre (Helmut Willke, Dirk Baecker, Friedrich Glasl), aus dem Institutionalismus (W. Richard Scott), der Verwaltungslehre (Andreas Ladner, Kuno Schedler) und aus praxisnahen, neueren Konzepten aus dem Bereich der öffentlichen Planung und Steuerung sowie der Netzwerktheorien (Herbert Schubert, Andrea Tabatt-Hirschfeldt, Klaus Grunwald, Klaus-Peter Ross, Urs Kaegi) mit einbezogen.

Der Professionalisierungsstand im Bereich der Sozialplanung ist im deutschsprachigen Raum sehr unterschiedlich weit. In Deutschland ist dieses Arbeitsfeld vor allem in den mittleren und grösseren Städten sehr ausgebaut und es besteht auch mit dem Verein für Sozialplanung ein fachlicher Zusammenschluss, der eigene Publikationen vorlegt. In der Schweiz ist der Begriff der Sozialplanung noch wenig etabliert und löst bei den staatlichen Stellen ganz verschiedene Assoziationen aus, wie eine Untersuchung aus Basel exemplarisch zeigt (Dittmann/Tappert 2015).

Die Autoren haben sich dazu entschieden, in diesem Buch den Begriff der Sozialwirtschaft zu verwenden, obwohl er in der Schweiz noch wenig bekannt ist. Bekannter sind hierzulande die Bezeichnungen «Sozialwesen» oder «Sozialbereich». Der Begriff des Sozialwesens geht auf die Ursprünge der vorprofessionellen Sozialen Arbeit zurück und umfasst alle Organisationen und Tätigkeiten im Fürsorgebereich, in der sozialen Wohlfahrt und der sozialen Sicherheit. Mit der zunehmenden Professionalisierung der Sozialen Hilfe, der Ausdifferenzierung der Angebote, der Institutionalisierung der Anbietenden und der erhöhten Zahl von 
staatlichen Versicherungssystemen bekam der Begriff klarere Konturen. Gleichzeitig wurde er immer mehr ausgeweitet, sodass sich seine Grenzen auflösten: Mit dem Begriff des Sozialwesens konnte die Gesamtheit dessen, was sich im Sozialen bildete, mehr oder weniger abgebildet werden. Vor über 25 Jahren erschien erstmals das «Handbuch des Sozialwesens Schweiz» (Fehlmann/Häfeli/Wagner 1987), herausgegeben von der Landeskonferenz für Sozialwesen, einem Zusammenschluss der grösseren Organisationen der Schweiz, der später aufgelöst wurde, da es sich als schwierig erwies, die verschiedenen Interessen der grossen und kleinen Organisationen in der Schweiz in einem Dachverband zusammenzuführen. Erst 2015 ist wieder ein neues Handbuch Sozialwesen Schweiz (Riedi/Meier Kressig/Benz Bartoletta/Zwilling/Aebi Zindel 2015) erschienen.

Etwas moderner klingt der Begriff «Sozialbereich», möglicherweise, weil der Begriff «Bereich» weniger fürsorglich klingt als der Begriff «Sozialwesen». Aber diese Bezeichnung ist im Grunde genommen ebenso weitgespannt und unpräzise, sodass sich daraus nur schwierig fachliche Definitionen bilden lassen.

Mit der Professionalisierung des Managements sozialer Einrichtungen und einhergehend mit einer umfangreichen Fachliteratur über das Sozialmanagement ist in der Folge - vor allem im deutschsprachigen Raum - auch der Begriff der Sozialwirtschaft aufgetaucht. Verwendet wurde er zuerst in Deutschland in den späten 1960er Jahren. Er wurde einer breiten Öffentlichkeit mit der Gründung der «Bank für Sozialwirtschaft GmbH» bekannt. Diese Bank erkannte die volkswirtschaftliche Bedeutung der Wohlfahrtsverbände und sah in diesem Bereich ein wichtiges und wachstumsorientiertes Geschäftsfeld. Ab den 1980er Jahren fand der Terminus «Sozialwirtschaft» als Übersetzung des französischen Begriffs «économie sociale» auch Eingang bei den europäischen Behörden. 2012 hat die EU ein umfangreiches Grundlagendokument, «Die Sozialwirtschaft in der Europäischen Union», verabschiedet (Europäischer Wirtschafts- und Sozialausschuss 2012) das nebst historischen und theoretischen Grundlagen auch Aussagen zur Bedeutung der Sozialwirtschaft unter dem Blickwinkel der «Strategie Europa 2020» macht. Auch wenn der Begriff der Sozialwirtschaft in der Schweiz noch neu ist, so ist er international weitgehend etabliert und wird in den meisten Studiengängen im Bereich des Sozialmanagements verwendet und gelehrt. Die Autoren haben sich nicht zuletzt deshalb dafür entschieden, den Begriff in diesem Buch aufzunehmen, um sich damit der internationalen fachlichen Diskussion anzuschliessen. Doch was ist unter der Sozialwirtschaft zu verstehen? Im Folgenden sollen einige zentrale Merkmale derselben erläutert werden.

Wird von der gängigen Unterscheidung ausgegangen, dass es einen privatwirtschaftlichen Sektor des Wirtschaftens gibt (Profit-Bereich mit Angebot und Nachfrage), einen öffentlichen Sektor (Staat) und einen Dritten Sektor (Non-Profit-Bereich), dann wird bereits deutlich, dass es zu kurz greift, die Sozialwirtschaft 
nur dem NPO-Sektor zuzuordnen, da viele Leistungen auch im öffentlichen Sektor erfolgen. Zunehmend treten nebst NPO und staatlichen Leistungserbringenden auch private, profitorientierte Anbietende auf dem Sozialmarkt auf. So stellt sich aus der Sicht der Leistungsbestellenden und -finanzierenden in den letzten Jahren zunehmend die Frage, ob neue soziale Dienstleistungen an den Staat, an die etablierten sozialen Organisationen oder eben an private Anbietende, die sich nicht als NPO definieren, vergeben werden sollen. Solche Überlegungen sind Ausdruck eines wirtschaftlichen Denkens, indem davon ausgegangen wird, dass Wettbewerb wichtig ist und dazu beiträgt, die Angebote kostengünstiger zu machen. Ob bei den Entscheidungen jeweils professionelle Kriterien oder der Preis den Ausschlag geben, ist eine schwierig zu beantwortende Frage. Sie verweist darauf, dass bei der Beurteilung von sozialen Dienstleistungen qualitative wie auch betriebswirtschaftliche Kriterien eine Rolle spielen. Mit dem Begriff der Sozialwirtschaft wird auf dieses Spannungsfeld hingewiesen, welches nach Erachten der Autoren nicht aufgelöst werden kann. Es ist ein zentrales Merkmal von und eine der wichtigsten Herausforderungen in sozialwirtschaftlichen Organisationen, dieses Spannungsfeld auszuhalten und sich nicht einseitig vom ökonomischen Denken leiten zu lassen, sondern die Arbeit immer daran messen zu lassen, ob es gelingt, soziale Dienstleistungen unter den Aspekten der Verfügbarkeit, Erreichbarkeit, Zugänglichkeit, Kontinuität, Qualität, Integrierbarkeit und Akzeptanz zu generieren. Dieser Anspruch an sozialwirtschaftliches Denken und Handeln «steht aus der Sicht der Disziplin und Profession der Sozialen Arbeit nicht zur Disposition», wie Grunwald (2011: 1557) in seinem Fazit über die Bedeutung der Sozialwirtschaft schreibt.

An dieser Situationsbeschreibung wird deutlich, dass - in Unterscheidung zum Begriff des Sozialwesens - mit der Bezeichnung «Sozialwirtschaft» ein Terminus zur Verfügung steht, der das Soziale mit dem Ökonomischen verbindet. So findet sich im Lexikon der Sozialwirtschaft folgende Definition:

«Die Sozialwirtschaft hat den sozialen und zugleich ökonomischen Zweck, individuelle und kollektive Wohlfahrt in Formen gemeinschaftlicher Selbstversorgung und öffentlich, freigemeinnützig oder gewerblich organisierter Versorgung zu bewerkstelligen. Diesen Zweck erfüllt sie in Interaktion von und mit Personen, die zur Bewältigung ihres täglichen Lebens Bedarf an einer spezifischen Versorgung haben. Sie wird, soweit sie nicht informell erfolgt, von Organisationen übernommen, die soziale Dienstleistungen anbieten» (Arnold/Maelicke 2008: 65).

Mit der Verwendung des Begriffs «Sozialwirtschaft» wird aber nicht nur deutlich, dass Anbietende sozialer Dienstleistungen nebst der fachlichen auch eine betriebswirtschaftliche Dimension beachten müssen, sondern es wird auch darauf aufmerksam gemacht, dass diese Leistungen eine «Wertschöpfung» erbringen. Nicht unwichtig ist dabei, zu erfassen, wie aus diesen sozialen Dienstleistungen letztlich 
auch ein bedeutender volkswirtschaftlicher Nutzen entsteht. Gerade diese beiden letzten Erkenntnisse sind eher neuere Sichtweisen, die unter dem Begriff «Social Return on Investment» (SROI) bekannt geworden sind. Die Bestimmung des SROI ist dabei ein Verfahren, die klassische ökonomische Bewertung von Investitionen (Return on investment) um einen sozialökonomischen, gesellschaftlichen und ökologischen Wert zu erweitern (Halfar/Moos/Schellberg 2014). Dieser Ansatz ist jedoch nicht nur für NPO, sondern auch für For-Profit-Organisationen zu verstehen und wird in diesem Bereich oft als "Corporate Social Responsibility» bezeichnet. Etwas verkürzt kann damit gesagt werden, dass das Soziale nicht immer unökonomisch und das Ökonomische nicht immer unsozial sein muss.

Es zeigt sich also, dass die Akteure der Sozialwirtschaft nicht darum herumkommen, sich mit verschiedenen Ansprüchen auseinanderzusetzen. Diese Ansprüche sind von Interessen, Funktionen und Rollenvorgaben gesteuert und so könnte von unterschiedlichen Logiken der jeweiligen Anspruchsgruppen gesprochen werden. Diese Logiken können in der Sprache von Niklas Luhmann (1987) als gesellschaftliche Funktionssysteme wie Politik, Wirtschaft, Medien und Soziale Arbeit bezeichnet werden. Für sozialwirtschaftliche Unternehmen bedeutet dies, dass sie diese unterschiedlichen Logiken der jeweiligen Stakeholder berücksichtigen müssen und nicht nur der wettbewerbsorientierten Handlungslogik des Markts folgen können. Für den Bereich der sozialpolitischen Steuerung - das Thema dieses Buchs - ist es deshalb von Bedeutung, sowohl die hierarchisch-gesetzesbezogene Steuerungslogik des Staats als auch die auf Solidarität und Gerechtigkeit beruhende Logik des NPO-Sektors zu berücksichtigen (Grunwald 2013).

Schliesslich soll darauf hingewiesen werden, dass die Sozialwirtschaft nicht nur die professionellen und formellen Leistungen von Organisationen umfasst, sondern auch die informellen Hilfeleistungen wie Freiwilligenarbeit, Selbsthilfe und die verschiedenen Formen zivilgesellschaftlichen Engagements mit einbezieht. Damit ist die Sozialwirtschaft auf drei verschiedenen Ebenen angesiedelt: 1) auf der Makroebene des sozialpolitischen und volkswirtschaftlichen Steuerns, 2) auf der Mesoebene der sozialwirtschaftlichen Organisationen und 3) auf der Mikroebene der Wohlfahrtsproduktion von Personen und Personengruppen jenseits organisatorischer Einbettung (Wendt 2010). 


\section{Folgerungen für zentrale Handlungskompetenzen der Systemgestaltung}

Aus diesen hier nur kurz ausgeführten Überlegungen haben sich die Autoren des vorliegenden Buches entschieden, den Begriff «Sozialwirtschaft» und nicht die Termini «Sozialwesen» oder «Sozialbereich» zu verwenden, um den Gegenstand und das Feld der sozialen Tätigkeiten zu bezeichnen. Es ist ihnen bewusst, dass die Begriffe «Sozialmanagement» und vor allem «Sozialwirtschaft» für einige Leserinnen und Leser noch neu sind; ebenso, dass das Wort «Wirtschaft» zur Befürchtung Anlass gibt, das Soziale werde dadurch unter das Diktat der Ökonomie gestellt. Die Buchautoren sehen diese Gefahr, teilen die Befürchtung aber nicht. Vielmehr sind sie davon überzeugt, dass die Auseinandersetzung mit Ökonomie und Betriebswirtschaftslehre eine Professionalisierung der Sozialen Arbeit zur Folge hat. Dies ist allerdings nur dann der Fall, wenn diese beiden Systeme miteinander kommunizieren.

Mit Blick auf die Handlungskompetenzen heisst dies, dass für das Management der eigenen Organisation, wie auch für die Systemgestaltung, die Rahmenbedingungen und die Besonderheiten der Sozialen Arbeit berücksichtigt werden müssen, damit der Austausch und das Verständnis zwischen den Systemen Soziales und Ökonomie auch wirklich stattfindet. Dies wird im vorliegenden Buch als «Sozialwirtschaftskompetenz» (siehe Abschnitt 3.4.1) bezeichnet.

\subsubsection{Management und Governance}

Eine weitreichende Form des Einbezugs der Zivilgesellschaft in Planungsprozesse ist in letzter Zeit unter dem Begriff «Governance» in verschiedenen Formen eingeführt worden und hat auch für die Sozialwirtschaft immer grössere Bedeutung erlangt. Allerdings ist auch dieser Begriff nicht eindeutig geklärt. "Grundmotiv des Governance-Diskurses ist die Frage, wie unterschiedliche Steuerungsmechanismen beziehungsweise -logiken ineinandergreifen (müssen), um ein bestimmtes Ergebnis zu erreichen» (Grunwald/Ross 2014: 26). Damit ergeben sich Veränderungen und neue Perspektiven der Steuerung, insbesondere indem die informellen Prozesse gegenüber den formellen an Bedeutung zunehmen: Selbstorganisation, Netzwerkbildung und Instabilität werden zu wichtigen Elementen der Steuerung. Grunwald und Ross (2014) unterscheiden denn auch zwei Perspektiven der Governance: eine analytische und eine normativ-strategische.

- $\quad$ Governance als analytisches Konzept ist ein Versuch, die zunehmende Unübersichtlichkeit und Überkomplexität in den Strukturen und Verfahren in 
Staat, Wirtschaft und den gesellschaftlichen Teilsystemen zu erfassen, aber auch die zunehmenden Verflechtungen und Interdependenzen zwischen a) der kommunalen, nationalen und internationalen Ebene, b) den verschiedenen Funktionssystemen, c) zwischen den Sektoren Staat, Markt, NPO und Primäre Netze im Wohlfahrtsmix und d) den unterschiedlichen sozialpolitischen Feldern zu analysieren. Alle diese Faktoren konstituieren den WelfareMix (Evers/Olk 1996) und verweisen auf die Problematik bei der Systemgestaltung.

- Zudem zeigt die Erfahrung, dass die sozialpolitische Steuerung dann stärker an ihre Grenzen stösst, je mehr versucht wird, vor allem über ihre Steuerungsmodi Macht und Hierarchie zu operieren. Ebenso werden die Grenzen sichtbar, wenn nur via Wettbewerb und Markt gesteuert wird. Die Systemgestaltung kann somit nicht nur durch politische Steuerung erfolgen, sondern sie muss auch aus einem Mix beziehungsweise einer Balance der Funktionslogiken und Werte der an einem Versorgungssystem beteiligten Akteure erfolgen. Die kollektive Findung der passenden Entscheidungsformen und damit die Gestaltung eines Entwicklungsprozesses sind ebenso wichtig wie die inhaltlich-fachliche Bestimmung der Arbeit. Governance passiert dann, wenn diese Unterschiedlichkeit anerkannt wird, wenn die verschiedenen Logiken aufeinander abgestimmt werden und wenn in diesem Sinn kooperativ und interaktiv gearbeitet wird.

- Governance als normatives und strategisches Konzept ist der Versuch, die oben beschriebene analytische Erkenntnis in Reformvorhaben umzusetzen. In der Politik- und Verwaltungswissenschaft wird Governance als unabdingbar für das Regieren moderner Gesellschaften verstanden (Papadopoulos in Grunwald/Ross 2014). In diesem Sinn wird das Konzept der «New Public Governance» als Reformkonzept der Verwaltungspolitik sowie als Alternative zum NPM verstanden. Das NPM habe eine übertriebene binnenorientierte und managerialistische Ausrichtung der Verwaltungspolitik (Jann/ Wegrich in Grunwald/Ross 2014) gebracht. Diese Kritik deckt sich mit der Beurteilung des NPM in der Schweiz. Auch hier haben die einseitig ökonomische und betriebswirtschaftliche Fokussierung der Verwaltungsarbeit sowie die übersteigerte Standardisierung und Systematisierung mindestens teilweise zu neuen bürokratischen Auswüchsen geführt, etwas, das gerade mit dem NPM hätte verändert werden sollen. Als positive Auswirkungen sind sicher die Trennung zwischen operativer und strategischer Ebene, die Idee der Leistungsvereinbarung und damit verbunden eine stärkere Zielformulierung und Leistungsüberprüfung zu nennen. 
Was sind nun aber die Besonderheiten der Entwicklung von «Management zu Governance»? Grunwald und Ross (2014) nennen sieben Punkte, die hier verkürzt aufgeführt werden:

(1) Governance fokussiert vor allem auf die interorganisationale Perspektive und damit auf die Beziehungen und Prozesse zwischen Organisationen und Gruppen von Akteuren.

(2) Governance setzt auf die gezielte Bildung und Pflege von sektorübergreifenden Politiknetzwerken als neue Institutionalisierungsform politischer Steuerung.

(3) Governance zielt damit explizit auf eine Kombination von Steuerungsformen («mix of modes») der verschiedenen gesellschaftlichen Teilbereiche (Formen davon sind z.B. Public-Private-Partnership oder Ko-Produktionen).

(4) Betonung des Stakeholder-Prinzips: Zivilgesellschaftliche Akteure sowie Betroffene sollen systematisch in die Problembewältigung einbezogen werden.

(5) Entscheidungsfindung soll durch Verhandlung und Beratung erfolgen und nicht durch Mehrheitsabstimmungen. Damit wird sich an einem konsensdemokratischen und nicht an einem konkurrenzdemokratischen Modell orientiert.

(6) Dienstleistungsorientierung und Effizienz - Ziele auch des NPM - sollen ergänzt werden durch weitere Ziele wie Stärkung von sozialer, politischer und administrativer Kohäsion und bürgerschaftlichem und politischem Engagement, womit die Exklusion bestimmter gesellschaftlicher Gruppen verhindert werden soll.

(7) Der Staat hat keine exklusive Verantwortung für sozialpolitische Steuerung und Koordination, sondern eher die Funktion einer «Systemverantwortung» oder eines «Interdependenzmanagements», was einer aktivierenden und kooperativen Politik entspricht.

Es soll nicht unterlassen werden, darauf hinzuweisen, dass es auch kritische Aspekte der Governance gibt. Gerade bei den oben beschriebenen Vorgehensweisen stellt sich die Frage nach der demokratischen Legitimation, da durch die kooperativen Formen und Netzwerkstrukturen die repräsentativ-demokratischen Entscheidungsformen umgangen werden können. Ferner besteht die Gefahr, dass auch in den Governance-Strukturen die schwachen Interessengruppen untergehen. Letztlich haben die Buchautoren noch nicht genügend Erfahrungen, um auch empirische Aussagen über die Wirkung der Governance-Konzepte machen zu können. Gleichwohl scheint es ihnen zentral, diesen Ansatz als Leitgedanken in die Systemgestaltung mitzunehmen. 


\section{Folgerungen für zentrale Handlungskompetenzen der Systemgestaltung}

Das Konzept des Managements und der Governance geht davon aus, die Vielfalt der Strukturen und Verfahren in Staat, Wirtschaft, Gesellschaft und den Funktionssystemen aufzunehmen und für die Bearbeitung und Lösung von sozialen Problemen nutzbar zu machen. Versorgungssysteme sind interorganisationale und interdisziplinäre Gebilde, in welchen verschiedenartige Logiken und Interessen wirken. Die Kunst der Gestaltung liegt darin, diese Systemakteure nicht nur zusammenzubringen, sondern auch für eine gemeinsame Arbeit zu gewinnen. Dies erfordert für die Systemgestaltung eine hohe Kompetenz zur Vernetzung und Kooperation (siehe Abschnitt 3.4.4), aber auch eine fundierte Handlungskompetenz zur Konzeptualisierung und Entwicklung von Projekten.

\subsubsection{Organisationales Handeln}

Da die Buchautoren, wie oben dargelegt, sozialpolitische Steuerung primär als eine Tätigkeit innerhalb des Funktionssystems Politik verstehen, ist ihrer Ansicht nach das Steuerungshandeln ganz wesentlich beeinflusst von der Organisation, in der die Systemgestaltung «angesiedelt» ist: also von den Strukturen der Verwaltung, die als primär bürokratisch geprägte Institution zu verstehen ist, sowie vom gesellschaftlichen und sozialpolitischen Umfeld, in dem sich die Hilfsangebote der leistungserbringenden Organisationen zu legitimieren haben. Auch wenn in letzter Zeit die Konzepte des NPM respektive des «Neuen Steuerungsmodells» wesentlichen Einfluss auf die Verwaltungseinrichtungen genommen haben (Schedler/Proeller 2006) und damit unternehmerische Elemente wie Konkurrenz und Wettbewerb, (Teil-)Privatisierung, Output- statt Inputorientierung, Kundenorientierung, die Trennung von strategischem und operativem Handeln, die Delegation von Verantwortung, Qualitätsmanagement und Wirkungsüberprüfung zu Leitgedanken für manche Reformen in der Verwaltung geworden sind, hat sich gezeigt, dass aus Verwaltungen nicht modernere Managementunternehmen entstehen. Die Bilanz dieser Bemühungen insgesamt ist eher ernüchternd (Hagn/ Hammerschmidt/Sagebiel 2012). Gewiss sind Strukturen und Abläufe oft «schlanker» geworden und eine stärkere Kundenorientierung hat das Bewusstsein in der Verwaltung dafür geschärft, dass sie nicht Selbstzweck ist, sondern im Dienste ihrer Benutzer/-innen zu stehen hat. Aber es ist auch festzustellen, wie Entscheide nun stärker nach einer ökonomischen Logik gefällt werden und dass Bemühungen um Qualität und Legitimation ihren Ausfluss in einer neuen Bürokratie gefunden 
haben. Verwaltungen lassen sich nicht einfach mit Managementtechniken verändern - Verwaltung bleiben, auch wenn sie sich wandeln, letztlich bürokratisch geprägte Institutionen.

Verwaltungshandeln vollzieht sich somit in einem widersprüchlichen Kontext: Einerseits ist es geprägt vom Selbstverständnis der Verwaltung als Dienstleisterin und andererseits ist die Verwaltung auch Auftraggeberin und Vollzugsorgan politisch gefällter sowie staatlich legitimierter Entscheide. In Anlehnung an Willke (1992) folgt die Steuerungslogik des Staats den Prinzipien des Rechts und der Macht. In diesem Sinn ist auch die Dienstleistungsorientierung der Verwaltung, die der Individualisierung und Kundenorientierung verpflichtet ist, in erster Linie von der Steuerungslogik des Staats geprägt. Verwaltungshandeln ist damit institutionelles Handeln, das von ritualisierten und habitualisierten Merkmalen gekennzeichnet ist.

Werden nun die Merkmale staatlich geprägten Handelns mit der Steuerungslogik von NGO und allenfalls sozialen Bewegungen verglichen, die Auftragnehmende der Verwaltung sind, so sind die strukturell bestimmten Reibungsflächen erkennbar. Im Abschnitt 3.2 wurde beim Thema Welfare-Mix das Sektorenmodell vorgestellt, in dem die unterschiedlichen Funktionslogiken und Werte der Sektoren erwähnt werden. Die Art der Sektoren respektive deren Institutionen bestimmen unter anderem auch die Logik der Kommunikation. Diese zu verstehen und für den jeweils andern Sektor verständlich zu machen, ist wohl eine der anspruchsvollsten professionellen Aufgaben bei der Systemgestaltung.

\section{a) Neo-Institutionalismus}

Der Neo-Institutionalismus (Senge 2006) bietet sich aus Sicht der Autoren dieses Buches an, um die strukturellen und institutionellen Rahmenbedingungen sowie ihre Auswirkungen auf das organisationale Handeln besser zu verstehen. Dieser Ansatz geht im Ursprung weit zurück bis auf den Bürokratieansatz von Max Weber (1972), wonach Bürokratien hauptsächlich durch folgende Merkmale gekennzeichnet sind: Arbeitsteilung und Befehlsgewalt, Amtshierarchie, Regelgebundenheit der Amtsführung und Aktenmässigkeit der Verwaltung. Diese vier Strukturmerkmale sind auch heute noch weitgehend konstituierend für Verwaltungen, auch wenn diese, wie oben erwähnt, sich zunehmend in einem widersprüchlichen Kontext (Nagel/Kessler/Sommerfeld 2007: 30) behaupten müssen.

Grundlegend für die Entwicklung des Institutionenbegriffs sind zwei grosse Theorierichtungen, die unterschiedliche Elemente betonen. Bei der einen Richtung geht es um die funktionalistische Theorietradition, die im amerikanischen Kontext durch Talcott Parsons (1986) und im europäischen insbesondere durch Emile Durkheim (1999) geprägt wurde. Gemeinsam gilt für diese und weitere Autoren, 
«dass Institutionen stets einen Beitrag zu anderen Gesellschaftsstrukturen und -prozessen leisten; Institutionen und Gesellschaft stehen also in einem funktionalen Zusammenhang» (Senge 2006: 37). Mit andern Worten: Institutionen sind immer funktional bezogen auf Gesellschafts- und Systemprozesse zu betrachten.

Die zweite zentrale Theorierichtung, welche die Konstruktion von Institutionen betont, kann als handlungstheoretische Fundierung bezeichnet werden. «In ihrem Mittelpunkt stehen nicht so sehr die grossen gesellschaftlichen Funktionssysteme wie Familie, Recht, Staat und so weiter», sondern vielmehr «der Prozess der Institutionalisierung. Das heisst: Es wird der durch Handlungen geschaffene, prozessuale Charakter von sozialen Strukturmomenten hervorgehoben» (Senge 2006: 37).

In einem sehr allgemeinen Verständnis lassen sich Institutionen im Kontext des Neo-Institutionalismus «als übergreifende Erwartungsstrukturen definieren, die darüber bestimmen, was angemessenes Handeln und Entscheiden ist» (Hasse/Krücken 2005: 5). Gemeint ist damit die handlungsleitende Kraft von Routine, welche für Verwaltungen bezeichnend ist, weil nicht primär die Individualisierung im Zentrum steht, sondern die Standardisierung und Normierung.

Die Autoren des vorliegenden Buches erachten das Konzept des Neo-Institutionalismus auch deshalb als gut geeignet für ihre Arbeit zur Gestaltung von Versorgungssystemen, da es zu den offenen Ansätzen zählt, die nicht die Organisation als autonome Einheit mit ihren internen Strukturen und Prozessen ins Zentrum stellt, sondern das Verhältnis von Organisation und Umwelt (siehe oben). Ganz bedeutsam für die Systemgestaltung ist zudem das Bewusstsein, dass Handeln aus der Sicht der Organisation zur Normierung neigt und aus der Sicht der Profession zur Individualisierung. Der Umgang mit diesem Spannungsfeld ist ein Merkmal des Handlungsverständnisses der Autoren, wie es im Abschnitt 3.5 näher beschrieben ist.

\section{b) Systemische Organisationsentwicklung}

Versorgungssysteme bestehen aus mehr oder weniger losen Verbünden sozialer Dienstleistungen, die in der Regel von Organisationen erbracht werden. Es spielt demnach eine Rolle, an welchem Organisationsverständnis sich die Systemgestaltung orientiert. Der theoretischen Grundausrichtung ihres Modells folgend, orientieren sich die Autoren auch beim Begriff der Organisation an einem systemtheoretischen Ansatz. In Abgrenzung zu einem eher «klassischen» Verständnis von Organisation, das davon ausgeht, dass sich eine Organisation als Ganzes erfassen, objektiv analysieren und in ihren Regeln und Strukturen beschreiben lässt (ähnlich einer Maschine), betont die systemische Perspektive vor allem die Wechselbeziehungen zwischen System und Umwelt der Organisation (Wimmer/Meissner/Wolf 
2009, Vahs 2007). Dieser Ansatz ist in Anlehnung an Dietmar Vahs (2007) durch folgende Merkmale charakterisiert: ganzheitliche und interdisziplinäre Sichtweise, Umweltorientierung, dynamische Betrachtung, Selbstregulierung respektive Selbstorganisation sowie Gestaltungsorientierung.

\section{Folgerungen für zentrale Handlungskompetenzen der Systemgestaltung}

Dieses in aller Kürze beschriebene Organisationsverständnis hat letztlich einen bedeutsamen Einfluss darauf, wie die handelnden Akteure der Systemgestaltung ihre Aufgaben in den einzelnen Phasen des Planungs- und Gestaltungsprozesses angehen, insbesondere dann, wenn es sich um die Zusammenarbeit mit den leistungserbringenden Organisationen handelt.

Da die Systemgestaltung immer im Auftrag einer (in der Regel staatlichen) Institution geschieht und die Akteure des Versorgungssystems ebenfalls als Vertreterinnen und Vertreter von verschiedenen leistungserbringenden Organisationen mitarbeiten, könnte auch von einer Art «Systemgestaltung in und mit Organisationen» gesprochen werden. Das Handeln aller Akteure im Versorgungssystem wird dabei in der Regel durch den Blickwickel der jeweiligen beteiligten Organisationen bestimmt. Eine zentrale Handlungskompetenz für die Systemgestaltung besteht deshalb darin, das Handeln der Personen immer im Kontext ihrer jeweiligen organisationalen Systeme zu sehen und Verständnis für unterschiedliche Logiken zu entwickeln. Als Moderierende des Versorgungssystems geht es aber auch darum, diese Unterschiedlichkeit zu vermitteln und für das Ganze nutzbar zu machen. In der Umsetzung ist dann ein prozessorientiertes Projektmanagement (siehe Abschnitt 3.4.5) eine weitere wichtige Handlungskompetenz.

\subsubsection{Kooperationsformen}

a) Intraorganisationale Kooperation auf staatlicher Ebene

Als intraorganisationale Kooperation auf staatlicher Ebene bezeichnen die Autoren die Zusammenarbeit der Steuerungsakteure innerhalb der Verwaltung respektive zwischen den verschiedenen Abteilungen und Departementen des politischen Systems. Die staatlichen Strukturen sind nach einer formalen, funktionalen Logik gegliedert (z.B. Bildung, Finanzen, Soziales, Gesundheit, Bau usw.). Soziale Probleme lassen sich inhaltlich kaum dieser Verwaltungsgliederung zuordnen. So betrifft zum Beispiel die Planung der kommunalen Alterspolitik mehrere Depar- 
temente: Soziales, Gesundheit, Bildung und Bau. Eine ganzheitliche und integrierte Systemgestaltung müsste also ämterübergreifend angegangen werden. Das heisst, es müssten zur Bearbeitung des Themas innerhalb der staatlichen Organe interdisziplinäre Kooperationen wie Projektgruppen oder Vernetzungssysteme aufgebaut werden, auch wenn das vorerst für Verwaltungen eher ungewöhnlich ist. Erfahrungen zeigen aber, dass in solchen Gremien dieser Prozess vielleicht etwas länger dauert, aber die Ergebnisse breiter mitgetragen werden und auch innovativere Lösungswege entstehen können.

\section{b) Interorganisationale Kooperation / Netzwerke}

Neben Wettbewerb bietet sich auch die Möglichkeit der Vernetzung und Kooperation sozialer Einrichtungen an. Der Impuls für Kooperation kann dabei von der sozialpolitischen Steuerung oder von den sozialen Einrichtungen ausgehen.

Im Sinn von Boris Holzer (2010: 163) ist mit Vernetzung eine Form kooperativer Praxis gemeint, die nicht zu einer Auflösung der kooperierenden Einheiten führt. Fokussiert werden die Entwicklung tragfähiger Kooperationsformen und die Gewährleistung von Handlungs- und Entscheidungsfähigkeit in gemeinsamen organisationalen Handlungskontexten. Kooperationen entstehen nicht von selbst, sondern sind im Rahmen eines gezielten Vorgehens zu entwickeln (Schubert 2008: 10ff.).

Eine erfolgreiche Netzwerkkooperation setzt ausreichende zeitliche, finanzielle und soziale Ressourcen sowie Kompetenzen der beteiligten Promotoren voraus. Um Kooperationen $\mathrm{zu}$ entwickeln und zu gestalten, ist eine vertrauensbasierte Kommunikation unter den beteiligten Akteuren eine zentrale Voraussetzung. Jörg Sydow und Arnold Windeler (2000: 122) heben in Kooperationsprozessen die Bedeutung sogenannter weicher Steuerungsmedien wie Vertrauen, Selbstverpflichtung der Akteure, Verlässlichkeit, Verhandlung und explizite oder implizite Kontrakte hervor.

Die Leitung sozialer Einrichtungen hat die Möglichkeit, die Gestaltung von Versorgungssystemen aktiv zu beeinflussen: Einerseits kann sie die Leistungen der Organisation gegenüber Mitbewerbenden und deren Angebote bewusst komplementär oder in Konkurrenz zu ihnen positionieren, anderseits kann sie mit anderen Organisationen eine Kooperation eingehen. 


\section{Folgerungen für zentrale Handlungskompetenzen der Systemgestaltung}

Auch wenn Erfahrungen zeigen, dass kooperativ gestaltete Prozesse zu befriedigenderen Ergebnissen führen als nicht kooperativ gestaltete, so ist doch auch zu erkennen, dass Kooperationen anspruchsvoll sind, weil sie einen Differenzierungsprozess bewirken. Das Gemeinsame, aber auch das Unterschiedliche der beteiligten Akteure wird sichtbar. Unterschiedliche Interessen können zu Konflikten führen, gleiche Interessen können Konkurrenzsituationen erzeugen. Für die Systemgestaltung sind Handlungskompetenzen wie Vernetzungen aufbauen oder Kooperationen unter den Beteiligten herstellen sehr wichtig. Eine hohe Empathie für die Situation der am Prozess Beteiligten und ein transparentes Vorgehen im Projektmanagement sind weitere zentrale Handlungskompetenzen. Natürlich ist nebst den methodischen Kompetenzen vor allem auch die Integrität der systemgestaltenden Person von besonderer Bedeutung.

\subsubsection{Innovation}

Anne Parpan-Blaser bezeichnet Innovation als Leitbegriff moderner Gesellschaften, ein Konzept mit einer beinahe schon penetranten Präsenz, wobei die Autorin eine Diskrepanz zwischen Innovationsdiskurs und sachlicher Analyse feststellt (Parpan-Blaser 2011: 13). Sie befasst sich denn auch mit der theoretischen und empirischen Grundlegung eines Konzepts zur Innovation in der Sozialen Arbeit, die für Versorgungssysteme wertvolle Bezugspunkte liefert. Laut Wolf Rainer Wendt (2005: 5) verlangen die Modernisierungsprozesse in Wirtschaft, Staat und Gesellschaft nach innovativen Lösungen. Hierbei versteht Wendt unter Innovation eine Neuerung beziehungsweise eine Veränderung, die eine Verbesserung bringt und sich durchsetzt. Matthias von Bergen (2015) findet in seiner Literaturrecherche zur Innovation im öffentlichen Sektor keine allgemeine Definition des Begriffs «Innovation», aber eine Vielzahl von auf sie hindeutenden Kategorien. Innovationen können zum einen unterschieden werden nach ihrer Art (Produkteinnovation, Serviceinnovation, Prozessinnovation usw.) und zum anderen nach dem Grad der Innovation (radikale Erneuerungen, Verbesserungsinnovation, Übernahme von anderswo bereits erfolgten Neuerungen usw.) (von Bergen 2015: 3). 
Nachfolgend werden Hinweise auf Innovationskonzepte im öffentlichen Sektor, in der Sozialwirtschaft und in der Sozialen Arbeit gegeben:

- Innovationskonzepte im öffentlichen Sektor: Innovationen im öffentlichstaatlichen Bereich beinhalten meist die Umsetzung neuer politischer Konzepte. Innovation wird hier nicht über Wettbewerbs- und Marktmechanismen getrieben, sondern durch politische Prozesse ausgelöst und beurteilt. Massgeblich sind der Handlungswille, die Finanzierungsmöglichkeiten sowie die sachlogische Bewertung und Evaluationsergebnisse einer Projektphase oder eines Modellversuchs (Zimmermann et al. in Parpan-Blaser 2011: 87). Von Bergen (2015) nennt - gestützt auf Forschungsergebnisse - folgende Erfolgsfaktoren: Problemwahrnehmung; engagierte, innovative Personen; Verwaltung, Politik und Leistungserbringende (Rolle von Schlüsselpersonen und Projektleiter/-in); das Suchen und Adaptieren von Lösungen; Ressourcen als Voraussetzungen (Bund, Kanton und / oder Gemeinde, aber auch Stiftungen, eigene Mittel der Leistungserbringenden); Kultur der Kooperation unter den Beteiligten; aktive und transparente Kommunikation; zeitlicher Aspekt: Zeitfenster nutzen; Zeit für die Verarbeitung von Veränderungen und deren Verankerung; oft auch zusätzliche Schlaufen; es würde nicht alles von heute auf morgen anders.

- Innovation in der Sozialwirtschaft: Wendt (2005) betont die Bedeutung von Sozialwirtschaft als Bedarfswirtschaft. Es könne also nicht darum gehen, einen Markt um neue Produkte zu bereichern, für die Abnehmende erst gefunden und beworben werden müssen. Zentral sei eine Nutzerorientierung, die zur individuellen und gemeinsamen Daseinsvorsorge beiträgt. Eine Angebotsorientierung würde zu einer teuren Über- und Fehlversorgung führen (Wendt 2005: 35). Die Herausforderung besteht nach diesen Überlegungen darin, die sich verändernden Bedarfe der Leistungsbeziehenden zu erkennen und die Angebote entsprechend zu gestalten. Daraus ergibt sich der Bedarf nach Innovation.

- Innovation in der Sozialen Arbeit: «Innovationen in der Sozialen Arbeit sind auf neuem oder neu kombiniertem Wissen basierende, in intendierten und kooperativen Prozessen entwickelte, neuartige Konzepte, Verfahren und Organisationsformen, die einen Mehrwert namentlich für Adressatinnen und Adressaten erzeugen» (Parpan-Blaser 2011: 242). In dieser Definition wird für Innovationen in der Sozialen Arbeit der Einbezug von Wissenschaft, professioneller Praxis sowie Klientinnen und Klienten vorausgesetzt. In ihren Ausführungen betont Parpan-Blaser (2011) die Plastizität von Innovation. 
Die Ebenen von Individuen (Mitarbeitende, Leitende, Klientinnen und Klienten), der Institution und Organisation, der Sozialen Arbeit als Disziplin und des gesellschaftlichen und politischen Umfelds sind einzubeziehen.

Innovationsfördernde Bedingungen können Folgende sein (siehe Stremlow/Riedweg/Da Rui/Müller 2016):

- $\quad$ Kultur der Kooperation unter den Beteiligten: Der Aufbau einer Kultur der Kooperation braucht Zeit und ist keine Selbstverständlichkeit, da die Beteiligten in einem Spannungsverhältnis von Kooperation und Konkurrenz zueinander stehen. Damit sich Vertrauen bilden kann, sind Offenheit und Transparenz wichtig.

- Innovative Schlüsselpersonen: Erfahrungen zeigen, dass wichtige Schlüsselpersonen in der öffentlichen Verwaltung, trotz bürokratischer Einengungen, wichtige Promotoren von innovativen Ansätzen sein können.

- Personelle und finanzielle Ressourcen: Innovation benötigt Mittel und ist nicht nebenbei zu machen. Der Einsatz entsprechender Ressourcen ist eine Investition in die Zukunft. Attraktive Rahmenbedingungen schaffen Anreize für neue Ideen.

- Zusammenarbeit mit Wissenschaft und Forschung: Aussenstehende Sichtweisen können durch Beratung Impulse für Innovation auslösen. Die Zusammenarbeit mit Wissenschaft und Forschung kann zudem den gedanklichen Horizont erweitern und Erfahrungen, die an andern Orten gemacht wurden, aufnehmen.

- Einbezug von Interessengruppen und Betroffenen: In vielen Themenbereichen, zum Beispiel in der Alterspolitik, existieren aktive Gruppierungen des zivilgesellschaftlichen Engagements. Betroffene selbst wie auch Vertretungen von Interessengruppen sind wichtige Akteure bei der Systemgestaltung, da ihnen die Möglichkeit gegeben wird, ihre Bedürfnisse direkt einzubringen. Zudem können diese Gruppen auch eine «Realitätsprüfung» neuer Ideen vornehmen.

\section{Folgerungen für zentrale Handlungskompetenzen der Systemgestaltung}

Die Beschreibung von innovationsfördernden Bedingungen und Promotoren zeigt, dass eine wichtige Aufgabe der Systemgestaltung darin besteht, die verschiedenen Akteure und Interessengruppen bis hin zu den direkt betroffenen Klientinnen und Klienten in einen verbindlichen Austausch- und Entwicklungsprozess einzubeziehen. Um das leisten zu können, braucht es eine grosse Offenheit, Bereitschaft und Fähigkeit, auf unterschiedliche Personengruppen zuzugehen und diese für die Idee 
der Zusammenarbeit bei der Systemgestaltung zu gewinnen. Wichtige methodische Handlungskompetenzen sind damit die Vermittlung und Vernetzung der Beteiligten und die Entwicklung von innovativen Projekten. Um das zu ermöglichen, ist eine hohe Prozesskompetenz (siehe Abschnitt 3.4.5) eine zentrale Fähigkeit der Systemgestaltung.

\subsubsection{Qualitätsentwicklung}

Zum Thema Qualitätsmanagement, Qualitätssicherung und Qualitätsentwicklung gibt es eine breite, schon kaum mehr zu überblickende Menge von Literatur. Allerdings bezieht sich diese fast ausschliesslich auf das Management einzelner Organisationen und kaum auf die Organisation im Kontext ihrer Umwelt. Noch weniger Literatur besteht zur Frage, welche Qualitätsanforderungen an die Systemgestaltung zu stellen sind. Die Autoren dieses Buches beziehen sich zu diesen Fragen auf Meinhold und Matul (2011), die ein ganzheitliches Qualitätsverständnis für die Soziale Arbeit formulieren und dabei die verschiedenen Ebenen der Qualität beschreiben, wobei sie sich insbesondere auch auf die Perspektiven der Ökonomie und der Sozialplanung beziehen. Hier werden auch die verschiedenen Logiken der Steuerung und deren Akteure aufgezeigt. Dieser Aspekt wurde im Rahmen dieses Buchs schon verschiedentlich erwähnt. In der untenstehenden Abbildung werden diese Ebenen und Logiken in der Übersicht dargestellt: 


\begin{tabular}{|c|c|c|c|c|}
\hline $\begin{array}{l}\text { Politische Ent- } \\
\text { scheidungsebene }\end{array}$ & $\begin{array}{l}\text { Volkswirtschaft- } \\
\text { liche Ebene }\end{array}$ & $\begin{array}{l}\text { Betriebliche } \\
\text { Ebene }\end{array}$ & $\begin{array}{l}\text { Ausführungs- } \\
\text { ebene der Fach- } \\
\text { kräfte }\end{array}$ & $\begin{array}{l}\text { Ebene der Kun- } \\
\text { denbeziehung }\end{array}$ \\
\hline \multicolumn{5}{|c|}{ Korrespondierende Anspruchsgruppen } \\
\hline Politik & Verwaltung & Leitungspersonen & Mitarbeitende & $\begin{array}{l}\text { Leistungs- } \\
\text { beziehende }\end{array}$ \\
\hline \multicolumn{5}{|c|}{ Aufgabe im Qualitätsmanagement } \\
\hline $\begin{array}{l}\text { Gesellschaftspoli- } \\
\text { tische Planung, } \\
\text { Institutionenwahl; } \\
\text { Gesetzgebung; } \\
\text { Subsidiarität; Re- } \\
\text { gelung des Zu- } \\
\text { gangs zu öffentli- } \\
\text { chen Leistungen }\end{array}$ & $\begin{array}{l}\text { Maximaler Nut- } \\
\text { zen mit geringst- } \\
\text { möglichem Auf- } \\
\text { wand; Steuerung } \\
\text { des Versorgungs- } \\
\text { systems; Gewähr- } \\
\text { leistung koordi- } \\
\text { nierter Dienste }\end{array}$ & $\begin{array}{l}\text { Verankerung des } \\
\text { Qualitätsmanage- } \\
\text { ments; Vereinba- } \\
\text { rung einer Quali- } \\
\text { tätspolitik } \\
\text { (Leitbild usw.); } \\
\text { Ressourcensteue- } \\
\text { rung, Wahrneh- } \\
\text { mung der Lei- } \\
\text { tungsfunktion }\end{array}$ & $\begin{array}{l}\text { Orientierung am } \\
\text { Bedarf der Nut- } \\
\text { zenden; fachliche } \\
\text { Standards verein- } \\
\text { baren und leben; } \\
\text { Rahmenbedingun- } \\
\text { gen sicherstellen }\end{array}$ & $\begin{array}{l}\text { Planung und } \\
\text { Durchführung von } \\
\text { individualisierten } \\
\text { Dienstleistungen }\end{array}$ \\
\hline \multicolumn{5}{|c|}{ Verschiedene Logiken } \\
\hline $\begin{array}{l}\text { Politische Steue- } \\
\text { rungslogik }\end{array}$ & Verteilungslogik & Managementlogik & Fachliche Logik & $\begin{array}{l}\text { Dienstleistungs- } \\
\text { logik }\end{array}$ \\
\hline $\begin{array}{l}\text { Legitimationsqua- } \\
\text { lität }\end{array}$ & Makroqualität & Mesoqualität & Mikroqualität & Servicequalität \\
\hline $\begin{array}{l}\text { Legalität der Sub- } \\
\text { ventionen; } \\
\text { Gleichbehandlung } \\
\text { der Leistungsad- } \\
\text { ressatinnen und } \\
\text {-adressaten; Leis- } \\
\text { tungsgerechtig- } \\
\text { keit; Sicherheit } \\
\text { und Kontinuität } \\
\text { des Angebots, } \\
\text { Effektivität der } \\
\text { erbrachten Leis- } \\
\text { tungen }\end{array}$ & $\begin{array}{l}\text { Auf Bedarf abge- } \\
\text { stimmtes Versor- } \\
\text { gungssystem; in- } \\
\text { stitutionelle } \\
\text { Abstimmung öf- } \\
\text { fentlicher und pri- } \\
\text { vater Leistungsan- } \\
\text { gebote; allokative } \\
\text { Effizienz der er- } \\
\text { brachten Leistun- } \\
\text { gen }\end{array}$ & $\begin{array}{l}\text { Steuerung durch } \\
\text { Zielvereinbarung; } \\
\text { Qualitätssiche- } \\
\text { rung und systema- } \\
\text { tische Verbesse- } \\
\text { rung; Controlling; } \\
\text { funktionales Lei- } \\
\text { ten; Effizienz und } \\
\text { Effektivität des } \\
\text { Mitteleinsatzes }\end{array}$ & $\begin{array}{l}\text { Qualität der ziel- } \\
\text { orientierten Wahr- } \\
\text { nehmung des Be- } \\
\text { darfs der } \\
\text { Leistungsbezie- } \\
\text { henden; Qualität } \\
\text { der handlungslei- } \\
\text { tenden Theorien } \\
\text { und Arbeitsprinzi- } \\
\text { pien; berufsethi- } \\
\text { sche Standards }\end{array}$ & $\begin{array}{l}\text { Zugänglichkeit, } \\
\text { Orientierung an } \\
\text { den Bedürfnissen } \\
\text { der Leistungsbe- } \\
\text { ziehenden; Quali- } \\
\text { tät der Zusam- } \\
\text { menarbeit mit } \\
\text { Nutzenden (Ko- } \\
\text { Produktion); Zu- } \\
\text { friedenheit der } \\
\text { Leistungsbezie- } \\
\text { henden }\end{array}$ \\
\hline
\end{tabular}

Abbildung 11: Ebenen der Qualitätsentwicklung

Quelle: Meinhold/Matul (2011) 
Dieses breite Verständnis von Qualität, in dem auch Widersprüche sichtbar werden, setzt voraus, dass sich Leistungsbestellende, Leistungsfinanzierende und Leistungserbringende eine gemeinsame Grundlage und Sichtweise gegenüber der Qualität der Leistungserbringung erarbeiten. Die Autoren machen nachfolgend einen ersten, pragmatischen Versuch, Qualitätsstandards für Versorgungssysteme und die für die Gestaltung verantwortlichen Instanzen zu formulieren:

Strukturqualität:

- Zugehörigkeit zum Versorgungssystem: Es ist geklärt, welche Einrichtungen zum Versorgungssystem gehören.

- Zielgruppe: Es ist klar, um welche Zielgruppe es sich für die Versorgung handelt.

- Sozialraum: Die räumliche Abgrenzung des Versorgungssystems ist bestimmt und begründet.

- $\quad$ Formalisierung: Zuständigkeiten und Entscheidungsverfahren sind festgehalten oder zumindest durch die Steuerungsinstanz offiziell ausgesprochen. Es bestehen formal einheitliche Leistungsvereinbarungen.

- Strategie: Die mittelfristige Strategie der sozialpolitischen Steuerung ist bekannt und diskutiert.

- Angebotspalette: Die Angebote im Versorgungssystem sind aufeinander abgestimmt und sozialräumlich auf die Zielgruppen bezogen aufgeteilt.

- $\quad$ Leistungsabgeltung: Leistungen für das Versorgungssystem im Auftrag der Steuerungsinstanz werden vergütet, sofern sie nicht Teil der Leistungsvereinbarung sind.

- Kommunikation: Es besteht Einsicht in die Leistungsaufträge der einzelnen Einrichtungen.

Prozessqualität:

- $\quad$ Im gesamten Planungs- und Entwicklungsprozess (Bedarf - Strategie - Angebotsentwicklung - Controlling) werden die dem Versorgungssystem zugehörigen Einrichtungen partizipativ einbezogen.

- $\quad$ Es finden in einem bestimmten Turnus Austausch- und Koordinationsgespräche statt, in denen auch über Perspektiven der Weiterentwicklung informiert wird und diskutiert werden kann.

- $\quad$ Es besteht eine gegenseitige Akzeptanz von fachlichen Abmachungen oder Regelungen.

- $\quad$ Eine kontinuierliche Reflexion des Leistungsangebots findet statt.

- Reportinggespräche zwischen Leistungsbestellenden und Leistungserbringenden umfassen betriebswirtschaftliche und fachliche Ergebnisse. 
Ergebnisqualität:

- $\quad$ Es besteht ein ausreichendes Leistungsangebot im Gesamtsystem.

- Die Leistungen sind institutionsübergreifend gut abgestimmt.

- $\quad$ Die Zahl der Überschneidungen und Schnittstellen in der Leistungserbringung ist minimiert.

- $\quad$ Die Zugänglichkeit der Nutzenden zum Leistungsangebot wird regelmässig geprüft.

- $\quad$ Versorgungslücken werden kontinuierlich erfasst.

\section{Folgerungen für zentrale Handlungskompetenzen der Systemgestaltung}

Die Qualitätssicherung und -entwicklung auf der Stufe von Versorgungssystemen aufzubauen, ist eine sehr anspruchsvolle Aufgabe, da es darum geht, eine Art von «Makroqualität» zu beschreiben. Qualität wird damit nicht auf der Ebene der einzelnen Organisation (das ist in der Regel schon vorhanden), sondern auf der Ebene des gesamten Versorgungssystems erfasst. Dies erfordert für die Gestaltung einerseits hohe konzeptuelle Kompetenzen, andererseits die Kompetenz, solche Projekte zu leiten und den Prozess mit unterschiedlichen Akteuren zu gestalten.

\subsection{Zentrale Handlungskompetenzen in der Systemgestaltung}

In den letzten beiden Abschnitten wurden wichtige Bezugskonzepte vorgestellt, die für die Gestaltung von Versorgungssystemen als relevant betrachtet werden. Die Erörterung dieser Aspekte hatte den Zweck, wichtige Handlungskompetenzen für eine Systemgestaltung in der Sozialwirtschaft abzuleiten. In diesem Abschnitt werden die fünf zentralen Handlungskompetenzen in einer Zusammenschau kurz beschrieben.

\subsubsection{Sozialwirtschaftskompetenz}

Indem die Sozialwirtschaftskompetenz als eine der fünf zentralen Handlungskompetenzen bezeichnet wird, wird zum Ausdruck gebracht, dass der Sozialbereich nicht ohne Verknüpfung mit der Ökonomie gesehen werden kann. Das äussert sich darin, dass vermehrt betriebswirtschaftliche Anforderungen an soziale Einrichtungen gestellt werden, zum Beispiel in Leistungsvereinbarungen, bei der Entwicklung von Qualitätsmerkmalen oder von Kennzahlen, die fachliche sowie ökonomische Standards beinhalten. 
Bei der Sozialwirtschaft verbinden sich fundierte Branchenkenntnisse des Sozialbereichs mit ökonomischem Wissen zu einem Gesamtblick auf sozialpolitische Themen und organisationale Aufgaben. Die Voraussetzung dafür sind spezifische Fachkenntnisse aus dem sozialen Bereich und ausgewählte Kenntnisse aus der Volkswirtschaft, der Betriebswirtschaft und der Verwaltungslehre. Gestaltungsfragen werden damit unter einem interdisziplinären Gesichtspunkt analysiert und bearbeitet. Diese Handlungskompetenz bezieht sich primär auf die Aneignung und Anwendung von Fachkompetenzen.

\subsubsection{Systemverständnis und Systemübersetzung}

Systemgestaltung zeichnet sich oft durch eine hohe Komplexität aus, die durch unterschiedliche Systemrealitäten oder potenzielle Widersprüchlichkeiten bei gleichzeitigen Abhängigkeiten geprägt ist. Daher sind das Verständnis für verschiedenste Systemlogiken sowie eine hohe Übersetzungs- und Vermittlungskompetenz eine wesentliche Handlungskompetenz.

Nebst analytischen Fähigkeiten ist besonders bedeutsam, dass sich die Verantwortlichen in verschiedene Systemrealitäten einfühlen können. Erst dadurch kann das Verhalten von beteiligten Akteuren auch als deren systembedingtes Rollenverhalten verstanden werden. Um diese Anforderungen erfüllen zu können, ist eine hohe Kommunikationskompetenz unabdingbar. So gilt es beispielsweise, dialogische Prozesse in Fachnetzwerken zu gestalten, anspruchsvolle fachliche Sachverhalte verschiedensten Akteuren verständlich zu erläutern und sich bei diesen Aufgaben mündlich und schriftlich gewandt ausdrücken zu können.

\subsubsection{Konzeptualisierungs- und Entwicklungskompetenz}

Diese Handlungskompetenz umfasst strategisch vorausschauendes, innovatives und zukunftsorientiertes Denken. Ebenso zentral sind analytische Fähigkeiten, um entsprechende Fachkonzepte zur Systemgestaltung entwickeln und verfassen zu können. Bei dieser Kernkompetenz ist unter anderem methodisches Know-how gefragt wie beispielweise:

- $\quad$ systematisches Analysieren von bestimmten Problemlagen und Aufgabenstellungen (z.B. Stärken- und Schwächenanalysen);

- Konzipieren und Durchführen von organisationsübergreifenden Strategieentwicklungen, Formulieren von Entwicklungszielen;

- Planen von gut realisierbaren Umsetzungsschritten; 
- Konzipieren von organisationsübergreifenden Qualitätsentwicklungskonzepten und -prozessen;

- Verfassen von Konzeptpapieren.

\subsubsection{Vernetzungs- und Kooperationskompetenz}

Mit der Fähigkeit zu Vernetzung und Kooperation wird auf eine spezifische Kompetenz verwiesen, verschiedenste Akteure mit ihren unterschiedlichsten Anliegen, Interessen und Leistungskapazitäten in produktive und verbindliche Formen der Zusammenarbeit zu bringen. Diese Handlungskompetenz umfasst zum einen methodisch-instrumentelle und prozessbezogene Fähigkeiten wie die Moderation von Vernetzungsprozessen mit einem breiten Spektrum von Beteiligten (politische Verantwortungsträger/-innen, Fachpersonen der Verwaltung, Leistungserbringende oder Vertreter/-innen der Zivilgesellschaft). Zum anderen sind Aushandlungs-, Mediations- und Konfliktlösungskompetenzen in Netzwerken bedeutsam. Ausserdem spielen bei dieser Gestaltungskompetenz wert- und haltungsbezogene Aspekte eine wichtige Rolle. Dies umfasst beispielsweise die Entwicklung einer Kultur gemeinsamer Verantwortung, in der sich die Beteiligten und Nutzenden als Ko-Produzierende von Leistungen verstehen. Ebenso schliesst dies die Fähigkeit ein, verbindliche Austauschbeziehungen zu schaffen, die auf einer gemeinsamen Vertrauensbasis gründen.

\subsubsection{Prozesskompetenz und Projektmanagement}

Systemgestaltung erfolgt meistens in Prozessen und in Form von Projekten. In diesem Kontext sind hohe Fähigkeiten vonnöten, in Prozessen zu denken und zu handeln. Da Prozesse selten linear verlaufen, auch wenn sie zielbezogen angelegt sind, ist es zentral, zwischen Ziel und Weg sowie zwischen dem Gewünschten und dem Möglichen zu unterscheiden und nicht zuletzt auch die Bedeutung von «Umwegen» zu erkennen. Prozessgestaltung verlangt viel Einfühlungsvermögen sowie eine hohe Sensibilität und Sorgfalt im Umgang mit den realen Gegebenheiten. Hilfreich sind dabei zudem Kenntnisse im Bereich des Changemanagements und des Innovationsmanagements.

Zur Prozesskompetenz gehört unter anderem Know-how im Projektmanagement. Dies umfasst die methodischen und technischen Fähigkeiten zur Planung, Durchführung und Auswertung von komplexen Projekten in einem Umfeld, das oft noch abgegrenzt und definiert werden muss. Hier sind mündliche und schriftliche kommunikative Fähigkeiten sowie Präsentationstechniken sehr bedeutsam. 


\subsection{Professionelles Handlungsverständnis}

Damit die oben ausgeführten Handlungskompetenzen zum Tragen kommen, wird ein entsprechendes Handlungsverständnis der mit der Gestaltung betrauten Akteure benötigt. In diesem Abschnitt werden verschiedene Aspekte des betreffenden Handlungsverständnisses beschrieben, die sich auf die Perspektive der Handelnden auf die Systemgestaltung, das Professionalitätsverständnis, Partizipation, Prozessentwicklung sowie Spannungsfelder und Dilemmata beziehen.

\subsubsection{Der Gestaltungsbegriff}

Wie bereits verschiedentlich ausgeführt, haben sich die Autoren dazu entschieden, anstelle der eher gebräuchlichen Begriffe «Planung» und «Steuerung» den Begriff «Gestaltung» zu verwenden (siehe Abschnitt 3.1). Sie sind einerseits überzeugt, dass das Planen von Prozessen wichtig ist, andererseits ist es ihnen aber auch bewusst, dass die vielfältigen Rahmenbedingungen, Einflussfaktoren oder die Beteiligung verschiedener Akteure den Prozessverlauf ebenso mitbestimmen - und dies zu Recht -, sodass die Kunst des Planens und Steuerns eher als ein Gestalten verstanden werden sollte.

Die Autoren möchten sich damit von einem technischen und linearen Verständnis von Handeln distanzieren und haben mit dem Begriff «Gestalten» ein Wort gewählt, das Handeln als etwas Interaktives, Prozessbezogenes und Partizipatives versteht. Sie sind sich nicht nur theoretisch, sondern auch praktisch der Grenzen bei der Steuerung von komplexen Systemen in der Sozialwirtschaft bewusst. Auch wenn sie bezüglich der Sache das Gleiche wie mit «Steuerung» und «Planung» meinen, so wollen sie ihr Handlungsverständnis mit dem Begriff des Gestaltens besonders verdeutlichen. Dies ist das Ziel dieses Abschnitts.

\subsubsection{Reflexive Professionalität}

Ohne hier näher auf grundsätzliche Fragen der Professionalisierung in der Sozialen Arbeit einzugehen, ${ }^{10}$ werden nachfolgend zentrale Merkmale professionellen Handelns aufgeführt, um daraus ein spezifisches Handlungsverständnis für die Systemgestaltung abzuleiten.

$10 \mathrm{Zu}$ diesem grundsätzlichen Diskurs zählt zum Beispiel die Frage, ob Soziale Arbeit überhaupt eine eigenständige Profession oder eher als «Semiprofession» zu verstehen sei oder ob Sozialarbeit / Sozialpädagogik eine eigene Wissenschaft sei. 
Soziale Arbeit wird zum überwiegenden Teil im Kontext und Auftrag von Institutionen geleistet. Unabhängig vom Handlungsbegriff und unabhängig von einem bestimmten Organisationsverständnis kann gesagt werden, dass Handeln nicht nur ein Ergebnis individueller Entscheidungsfindung oder interaktiver Prozesse ist, sondern ganz wesentlich durch die institutionellen Rahmenbedingungen mitbestimmt wird (Senge 2006).

Zum professionellen Handeln gehören ein spezifisches, auf den Gegenstand des Handelns bezogenes Fachwissen sowie eine spezifische Methodologie mit den entsprechenden Kompetenzen. Auf methodologischer Ebene muss geklärt werden, welche Funktion von Handeln im Zentrum steht. In einer gängigen Unterscheidung wird in der Sozialen Arbeit von einer präventiven / vorbeugenden, einer wiederherstellenden und einer entwickelnden Funktion gesprochen. Entsprechend der Funktion sind bestimmte Handlungskompetenzen von Bedeutung (z.B. Beratungskompetenz bei der wiederherstellenden Funktion). Wenn in der Literatur der Sozialplanung von «Steuerung», «Planung» oder «Gestaltung» gesprochen wird, so sind dies Handlungen, die erstens nicht typisch sozialarbeiterische Kompetenzen umfassen und zweitens Kompetenzen betreffen, die mehrere Professionen für sich beanspruchen. Begrifflich wird «Steuern» einerseits dem Bereich des Managements und andererseits dem Bereich der Verwaltung zugeschrieben - nicht aber der Sozialen Arbeit. Sozialpolitische Steuerungsaufgaben, zum Beispiel die Konzeptualisierung und Gestaltung von Versorgungssystemen, waren denn auch lange Zeit keine Aufgabe der Sozialen Arbeit, sondern eher eine Verwaltungstätigkeit. Dementsprechend sind auch nur wenige Fachleute aus der Sozialwirtschaft an diesen Stellen anzutreffen; viel eher sind Juristinnen und Juristen, Soziologinnen und Soziologen oder Verwaltungsfachkräfte zu finden, die diese Funktionen übernehmen.

Aus der Sicht einer systemtheoretischen Professionstheorie handeln Professionelle zudem ausschliesslich in der Logik des jeweiligen Funktionssystems. Wird nun über die Professionalisierung sozialpolitischer Steuerung gesprochen, so ist dabei zu berücksichtigen, dass sich dieses Handlungsfeld im Schnittpunkt mindestens zweier Funktionssysteme abspielt: des Funktionssystems Politik und des Funktionssystems Soziale Arbeit. Zudem kommt dem Funktionssystem Recht in der Anbindung an die Politik eine besondere Bedeutung zu. Wenn es also um Fragen geht, die die Interaktion zwischen Funktionssystemen betreffen, so ist das Modell der dezentralen Kontextsteuerung von Willke (2000) hilfreich. ${ }^{11}$ In seiner Aufbereitung der Systemtheorie (und in Abgrenzung zu Luhmann) treten bei ihm Systeme auch als Akteure auf. Das heisst im Fall des vorliegenden Modells, dass

11 Das Modell der dezentralen Kontextsteuerung und wie Systeme als Akteure auftreten, ist in Willke (2000) beschrieben. Konstantin Bähr (2001) hat diese Akteurstheorie aufgenommen und auf den Bildungsbereich angewandt. 
das Funktionssystem Politik mit dem Funktionssystem Soziale Arbeit beziehungsweise deren Organisationen, «verhandelt» und sie «steuert». Dies ist allerdings nur möglich, wenn sich die «Steuerungsakteure» des Funktionssystems Politik auch auf die Logiken des Funktionssystems Soziale Arbeit einlassen und damit vertraut werden.

\subsubsection{Partizipation}

Partizipation, verstanden als Einbezug und Mitwirkung von Klientinnen und Klienten und ihrem Umfeld, entspricht aus der Sicht der Sozialen Arbeit einer alten Tradition. Beratung und Unterstützung gelingen nur dann nachhaltig, wenn sich die Betroffenen nicht nur als Empfänger/-innen, sondern auch als Ko-Produzierende der Hilfeleistung verstehen. Was für die Einzelnen und Gruppen gilt, hat auch - in anderen Formen - bei Prozessen zur Systemgestaltung Gültigkeit. Auch hier gilt das grundsätzliche Prinzip, die Mitwirkung von Individuen, Personengruppen, Bewegungen, Interessenvertretungen und Organisationen im Kontext der jeweiligen Aufgabe in einem Planungs- und Gestaltungsprozess nach Möglichkeit mit einzubeziehen.

Wie bereits erwähnt, sind bei der Systemgestaltung die primären handelnden Institutionen einerseits staatliche (z.B. die Verwaltung) und andererseits Institutionen der Sozialwirtschaft. Bereits in diesem Verhältnis zwischen Leistungsbestellenden und Leistungserbringenden zeigt sich die Grundhaltung der Arbeitsbeziehung. Wenn die leistungserbringenden Organisationen lediglich Empfängerinnen von Aufträgen sind und die staatlichen Organe die Leistungen definieren und Aufträge erteilen, ohne diese mit den Organisationen der Sozialwirtschaft auszuhandeln, zu klären und die Qualitätsstandards gemeinsam festzulegen, dann entwickelt sich als Grundmuster ein hierarchisches Arbeitsverhältnis von oben nach unten und versetzt die Leistungserbringenden in eine einseitige Abhängigkeit. Dieses Grundmuster stipuliert dann Leistungsverträge und nicht Leistungsvereinbarungen. Ohne die unterschiedlichen Funktionen von Auftragserteilung und -empfangenden zu verwischen, lässt sich durchaus ein partizipatives Vorgehen in der Ausgestaltung des Leistungsangebots verwirklichen. Dies ist nicht zuletzt auch deshalb angezeigt, weil die fachlichen Kompetenzen und insbesondere die Erfahrungen stärker bei den leistungserbringenden Organisationen liegen als bei den leistungsbestellenden. Zudem sind bei sozialplanerischen Prozessen weitere Stakeholder wie Interessenverbände, Fachorganisationen, Einrichtungen mit Bezug zum System wie auch interessierte oder betroffene Bürgerinnen und Bürger im entsprechenden Sozialraum zu berücksichtigen. Partizipation wird so zu einem 
Arbeitsprinzip und zu einer prägenden Grundhaltung bei der Gestaltung von Planungsprozessen.

Gemäss Heike Walk (2011) können drei Funktionen von Partizipation unterschieden werden:

(1) «Die demokratische Funktion von Partizipation umfasst sowohl die Forderung nach einer verbesserten und verstärkten Mitsprache an politischen Entscheidungen von Seiten der Zivilgesellschaft als auch die Erhöhung der Akzeptanz und somit Legitimierung von Prozessen und Entscheidungen durch eine verstärkte, gegebenenfalls auch repräsentative Einbeziehung unterschiedlicher Interessen (...)» (Walk 2011: 63).

(2) «Die ökonomische Funktion richtet sich vor allem auf die Effizienz von Entscheidungsprozessen. Dabei wird argumentiert, dass Partizipation einer verbesserten Kommunikation zwischen den Beteiligten dient und zur Berücksichtigung verschiedener Interessen beziehungsweise Bedürfnisse sowie in der Folge zu einer bedürfnisgerechteren Planung führt und dadurch nicht zuletzt nachhaltigere Entscheidungen und auf die Dauer weniger kostenintensive Umsetzungen generieren werden» (Walk 2011: 64).

(3) «Die emanzipatorische Funktion von Partizipation nimmt die politische Handlungskompetenz und das Selbstbewusstsein der Bürger in den Blick. Durch vermehrte Partizipation soll der Benachteiligung einzelner, bisher ausgegrenzter Bevölkerungsgruppen und Positionen entgegengewirkt werden. Weiterhin wird davon ausgegangen, dass gesellschaftliche, private und wirtschaftliche Akteure zu eigenverantwortlichem Handeln motiviert, unterschiedliche Lernprozesse in Gang gesetzt sowie langfristig tragfähige Strukturen entwickelt werden (...)» (ebd.).

Partizipation ist nicht Selbstzweck, sondern hat das Ziel, die Qualität und die Legitimität politischer Entscheide zu gewährleisten und zu verbessern. Das Prinzip der Partizipation wird dabei auch durch die demokratiepolitischen Rahmenbedingungen und die gewachsene Mitwirkungskultur des jeweiligen Sozialraums bestimmt. Partizipation in Planungsprozessen prägt gleichzeitig diese Kultur. Professionelle, partizipative Vorgehen und Methoden müssen demnach einerseits den jeweiligen Kontext kennen und berücksichtigen und andererseits aber auch neue Wege aufzeigen, wie die Mitbeteiligung weiterentwickelt und umgesetzt werden kann. Nicht nur in der Schweiz mit ihren ausgeprägten direktdemokratischen Mitbestimmungsrechten und ihrem konsensorientierten Demokratieverständnis hat die Partizipation einen hohen Stellenwert, auch in andern demokratischen Ländern (z.B. Deutschland, Grossbritannien) ist ein Diskurs für mehr politische Mitbeteiligung im Gang (Hebestreit 2013). Dabei hat sich auch gezeigt, dass die bisherigen 
formalen Beteiligungsrechte und die klassische Bürgerbeteiligung an Grenzen stossen (Hebestreit 2013: 87). Als Antwort darauf sind unter dem Begriff «diskursive Beteiligungsverfahren» (Nanz/Fritsche 2012) neue partizipative Formen entwickelt und erprobt worden, in denen Bürgerbeteiligung als kommunikativer Prozess verstanden wird.

\subsubsection{Prozessorientierung}

Gestaltendes Handeln geschieht immer auch als Prozess. Dies ergibt sich schon aus der Tatsache, dass bei der Steuerung deutlich einzelne Phasen unterschieden werden können wie Situationsanalyse, Strategieentwicklung, Umsetzung, Controlling, Aktualisierung der Problemsituation und so weiter. Aber nicht nur die Abfolge der Phasen kann als Prozess verstanden werden, sondern auch die Gestaltung der einzelnen Phasen ist von einem Prozessverständnis geprägt. Gemeint ist damit ein interaktives und iteratives Vorgehen, indem das eigene und fremde Handeln ständig im Hinblick auf seine Wirkung reflektiert wird.

In der Sozialen Arbeit sind Interaktion und Kommunikation die zentralen «Steuerungsmittel» im Umgang mit den verschiedenen Anspruchsgruppen. Bei der Systemgestaltung gilt dies auch, mit dem Unterschied allerdings, dass die steuernde Instanz als Auftraggeberin über die Mittel und die Macht zur Auftragserteilung verfügt. Dies hat mit den oben erwähnten institutionellen Rahmenbedingungen zu tun. Es ist deshalb wichtig - und ein Teil professionellen Handelns -, sich dieser Situation bewusst zu sein. Die Prozessgestaltung geschieht deshalb nicht hierarchisch, sondern heterarchisch. Diese Unterscheidung zeigt sich oft in der Begrifflichkeit, zum Beispiel darin, ob von einem Leistungsauftrag oder einer Leistungsvereinbarung gesprochen wird. Zur Prozessgestaltung gehört damit auch die Fähigkeit, rollenbewusst und rollenadäquat zu handeln.

\subsubsection{Umgang mit Spannungsfeldern und Dilemmata}

In verschiedener Hinsicht haben die Autoren in diesem Buch immer wieder auf Spannungsfelder, Widersprüchlichkeiten, Dilemmata oder gar Paradoxien hingewiesen, die sich beim Versuch, komplexe Versorgungssysteme in einem sektoriellen Feld mit unterschiedlichen Funktionslogiken und Werten zu steuern, ergeben. Es existieren zwar Regeln, Techniken und Instrumente, die zur Bearbeitung einzelner Aufgaben dienlich sind. Aber die Handlungsweise in der Systemgestaltung ist geprägt von vielen Prämissen und Faktoren, die nicht nur Klarheiten und Rationalitäten erzeugen, sondern ebenso sehr Unberechenbarkeiten und Dilemmata. 
Es sind inzwischen auch bereits Konzepte des Managements von Dilemmata und Paradoxien formuliert worden (Grunwald 2013), die davon ausgehen, dass Erklärungen für organisationale Strukturen und Prozesse, die einseitig nur auf Rationalitäten beruhen, immer unvollständig sind. Deshalb kann der Umgang mit Spannungsfeldern als eine zentrale Aufgabe im Sozialmanagement angesehen werden.

Der Umgang mit paradoxen Anforderungen ist aber nicht nur bei der Systemgestaltung von Bedeutung, sondern kann geradezu als konstituierendes Merkmal der Arbeit von NPO verstanden werden (Simsa 2002). Die betreffende Autorin weist darauf hin, dass nicht nur intermediäre Funktionen - wie die Systemgestaltung auch verstanden werden kann - sondern ganz grundsätzlich NPO besonderen Widersprüchen ausgesetzt seien, was Forschungsresultate aus verschiedenen Ländern zeigen würden. Gründe dafür seien die vielfältigen und unterschiedlichen gesellschaftlichen Ansprüche an NPO und die zum Teil divergierenden Interessen, die damit verbunden sind. Viele Dilemmata seien fundamentale Widersprüche, die nicht einseitig gelöst werden könnten, jedoch für die Leitungspersonen besondere Kompetenzen wie beispielsweise Konfliktmanagement erfordern würden.

Auf das Spannungsfeld von Standardisierung versus Individualisierung, welches Bezug auf das Thema organisationales Handeln im Abschnitt 3.3.3 nimmt, soll im Folgenden etwas näher eingegangen werden. Bürokratien sind von ihrer Funktionslogik her Organisationen, die zur Normierung und Standardisierung tendieren und die Lösung von Problemen im Prinzip von «mehr desselben» sehen. Die Profession der Sozialen Arbeit kennt das Handlungsprinzip «Individualisierung», womit gemeint ist, dem Einzelfall gerecht zu werden. Dieser Grundsatz gilt vorab für die Hilfe an der beziehungsweise am Einzelnen, lässt sich aber auch auf Gruppen, Organisationen und Gemeinwesen übertragen. Dieses Spannungsfeld besteht auch in der Systemgestaltung. Wichtig scheint, dieses in der Arbeit zu erkennen und nicht zu verneinen, sondern die unterschiedlichen dahinterstehenden Prinzipien und Werte als besondere Herausforderung bei der Suche nach Lösungen anzuerkennen.

In diesem Kapitel wurden die Kontextbedingungen, methodischen Annahmen und zentralen Kompetenzen zur Systemgestaltung betrachtet. Diese Überlegungen fliessen bei den folgenden Ausführungen zu den Aufgaben der Systemgestaltung (Kapitel 4) ein. Insbesondere wird dabei dem Gestaltungsprozess Rechnung getragen. 
Open Access Dieses Kapitel wird unter der Creative Commons Namensnennung 4.0 International Lizenz (http://creativecommons.org/licenses/by/4.0/deed.de) veröffentlicht, welche die Nutzung, Vervielfältigung, Bearbeitung, Verbreitung und Wiedergabe in jeglichem Medium und Format erlaubt, sofern Sie den/die ursprünglichen Autor(en) und die Quelle ordnungsgemäß nennen, einen Link zur Creative Commons Lizenz beifügen und angeben, ob Änderungen vorgenommen wurden.

Die in diesem Kapitel enthaltenen Bilder und sonstiges Drittmaterial unterliegen ebenfalls der genannten Creative Commons Lizenz, sofern sich aus der Abbildungslegende nichts anderes ergibt. Sofern das betreffende Material nicht unter der genannten Creative Commons Lizenz steht und die betreffende Handlung nicht nach gesetzlichen Vorschriften erlaubt ist, ist für die oben aufgeführten Weiterverwendungen des Materials die Einwilligung des jeweiligen Rechteinhabers einzuholen.

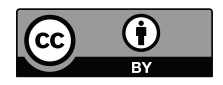

